\title{
Regular Tensor Algebras
}

\author{
By
}

\author{
Daniel A. Dubin* and Mark A. Hennings**
}

\begin{abstract}
A convolution algebra is a normal sequence space $\lambda$ that is closed under the convolution product. Let $E$ be a nuclear Fréchet locally convex space, and let $p=\left\{p_{r}: r \in N \cup\{0\}\right\}$ be a family of Hilbertian seminorms defining its topology. We define the vector space $T(E, \lambda, p)=$ $\left\{\left(x_{n}\right) \in \mathrm{I}_{n \geqq 0} \hat{\otimes}^{n} E:\left(\otimes_{\sigma}^{n} p_{r}\left(x_{n}\right)\right) \in \lambda, r \geqq 0\right\}$, equipped with the topology obtained from the seminorms $\sum_{n \geqq 0}\left|u_{n}\right| \bigotimes_{\sigma}^{n} p_{r}$ where $r \geqq 0$ and $u \in \lambda \times$, the Köthe dual of $\lambda$. For certain sequence spaces, the resulting space does not depend on $p$ and we write $T(E, \lambda)$. Such is the case for sequence spaces of type $h$.

Certain properties of $\lambda$ transfer directly to $T(E, \lambda, p)$. In particular, if $\lambda$ is complete (respectively Fréchet, a topological algebra), then so is $T(E, \lambda, p)$. A regular tensor algebra is a space $T(E, \lambda)$ for $\lambda$ a perfect topological convolution algebra with jointly continuous product which is of type $h . T(E, \lambda)$ is then nuclear, and reflexive if Fréchet.

We examine the topological properties of the spaces $T(E, \lambda, p)$. Other than invertibility, these are the same as for $E_{\otimes}=\bar{\bigoplus}_{n \geqq 0} \otimes^{n} E$. We then consider the order properties arising from a natural involution. The positive wedge $K(\lambda)$ is always a proper strict- $b$ cone, and if $\lambda$ is of type $h$ and $\lambda \subseteq h$, its closure is proper. Here $h$ is the sequence space isomorphic to the space $H(\boldsymbol{C})$ of entire functions. In particular, $T(E, h)$ is a regular tensor algebra which is locally multiplicatively convex and whose closed cone is proper. Finally, we present three conditions which are sufficient for $K(\lambda)$ to be normal.
\end{abstract}

\section{$\S 1$. Introduction}

In multilinear algebra and differential geometry, the contravariant tensor algebra of a vector space is of some importance. Studying Wightman's formulation of relativistic quantum field theory [9], [26], [29], Borchers [5], [6] and Uhlmann [28] independently obtained a reformulation based on a topological generalisation of a contravariant tensor algebra. With $E=S(R)$, the space of smooth functions of rapid decrease, they considered $E_{\otimes}=\bigoplus_{n \geqq 0} \bar{\otimes}^{n} E$ as a topological *-algebra. Our notation is that $\bar{\otimes}^{n} E$ is the $n$-fold tensor product of $E$

Communicated by H. Araki, February 16, 1989.

* The Open University, Walton Hall, Milton Keynes, MK7 6AA.

** Sidney Sussex College, Cambridge, CB2 3HU. 
with itself, completed in the inductive tensor product topology. The sum is meant to be the locally convex direct sum.

The theory of non-normed topological algebras is rather undeveloped, particularly in contrast with the theory of $C^{*}$-algebras. As Borchers [6] points out, so little was known about such matters that no interest was shown in this algebra until the work of Wyss [30], [31], in 1958. For the last decade and a half some progress in the analysis of $E_{\otimes}$ has been achieved, but the basic problem remains unsolved: in order to prove the existence of nontrivial relativistic quantum fields, one must prove the existence of nontrivial positive functionals on $E_{\otimes}$ which annihilate certain ideals.

Now certain other physical systems are, in many respects, similar to relativistic quantum field theory, notably nonrelativistic quantum statistical mechanics, and the so-called current algebras. In [4], Alcantara and one of us (D.A.D.) were able to show that these systems could also be described by the algebra $E_{\otimes}$ by choosing different spaces $E$. These considerations lend further support for more study of $E_{\otimes}$ and its quotient algebras $E_{\otimes} / I$, where $I$ is a complemented *-invariant positive ideal. Such quotient algebras are characteristic examples of $I^{*}$-algebras [4], and Alcantara has even shown that every $I^{*}$-algebra is a quotient algebra of this sort [3].

At the risk of oversimplification, the underlying obstruction to proving the existence theorems of the sort needed for applications is positivity and the relation between the order properties of $E_{\otimes}$ and $E_{\otimes} / I$. With this in mind, Lassner [18] and Yngvason [32] have considered alternative topologies on $E_{\otimes}$ better suited to the study of the order properties than the original. Subsequently Hofmann [12], [13], [14], [15] and Schmüdgen [24], [25] further analysed these topologies on $E_{\otimes}$.

Given a topology on $E_{\otimes}$, coarser than the original, with respect to which $E_{\otimes}$ is a locally convex algebra, one might consider the completion in this topology. The resulting space will not necessarily be an algebra, but it can be. This is the theme of this work. We consider certain topologies on $E_{\otimes}$ which lead to complete algebras.

In so doing, we were guided by the similarity in form between the product on tensor algebras and the convolution product of infinite series. This has led us to consider topologies for $E_{\otimes}$ defined by certain sequence spaces. It has proved possible to transfer a number of properties from these sequence spaces to the tensor algebras. Some properties, such as nuclearity and metrisability, transfer directly. Others only transfer indirectly, particularly the order pro- 
perties. This has led us to isolate certain properties of sequence spaces with a view to obtaining useful classes of tensor algebras.

In the next section we shall consider sequence spaces. After establishing some notation and terminology, we recall certain results concerning sequence spaces which will be of use subsequently. With a view towards properties of tensor algebras, we have introduced the class of sequence spaces that we have termed convolution algebras. As is well-known, the sequence space $l_{1}$ is stable under Cauchy's convolution product. More generally, a sequence space stable under the convolution product is called a convolution algebra.

In $\S 3$, for each convolution algebra $\lambda$ we construct the tensor algebra $T(E, \lambda)$ over a nuclear Fréchet space $E$ and consider its topological properties. Following that, we consider the algebraic and order properties of tensor algebras in $\$ 4$. Our principal conclusions are these. We define a regular tensor algebra to be a tensor algebra which is a complete nuclear topological algebra with jointly continuous product, and which is independent of the choice of seminorms for $E$. For each such $E$, there exist uncountably many regular tensor algebras. There even exist uncountably many with normal positive cones, once we have introduced an involution to the algebra. A complete analysis of the relation between the properties of $\lambda$ and the propriety or normality of the closed positive cone $\overline{K(\lambda)}$ of $T(E, \lambda)$ remains open. We do however have several sufficiency conditions for $\lambda$ in order that $\overline{K(\lambda)}^{\lambda}$ be proper or normal.

We have also found $\lambda$ such that $T(E, \lambda)$ is locally multiplicatively convex, and there exists one $\lambda$ such that $T(E, \lambda)$ is locally multiplicatively convex, Fréchet and regular.

One of us (D.A.D.) is pleased to acknowledge talks with Gerald Hofmann who convinced him of the interesting possibilities inherent in these exotic topologies, and with Jim Clunie who was helpful on a number of occasions, particularly with regard to convolution algebras. It is a pleasure to acknowledge a conversation with Konrad Schmüdgen, who suggested consideration of the left regular representation, and the second author (M.A.H.) would like to thank Dr. M.J.C. Gover for some interesting ideas concerning sequence spaces of type $p$.

\section{$\S 2$. Sequence Spaces and Convolution Algebras}

Sequence spaces were first introduced by Grothendieck [11] to provide the simplest examples of nuclear locally convex spaces. Many of their properties 
have been studied by Köthe [17], Garnir et al. [10] and others. Since the results and notation concerning sequence spaces will be fundamental to our theory we shall, in the next few sections, summarise these facts for later use. Where proofs have been omitted, they can be found in Köthe [17].

\section{$\S 2 \cdot 1$. General definitions}

Definition 2.1. (a) denote by $\omega$ (respectively $\phi$ ) the space of all complex sequences $a=\left(a_{n}\right)=\left(a_{0}, a_{1}, \ldots\right)$ (respectively which are eventually zero);

(b) a basis for $\phi$ consists of the sequences $\{e(n): n \geqq 0\}$, where

$$
e(n)_{k}=\delta_{n k} \quad n, k \geqq 0 \text {; }
$$

(c) a sequence space $\lambda$ is a subset of $\omega$ containing $\phi$ which is a complex vector space with respect to pointwise operations;

(d) a sequence space $\lambda$ is normal if, whenever $x \in \lambda$ and $y \in \omega$ satisfy $\left|y_{n}\right| \leqq\left|x_{n}\right|$ for all $n \geqq 0$, we have $y \in \lambda$;

(e) if $A \subseteq \omega$ is such that for any $n \geqq 0$ we have $a \in A$ with $a_{n} \neq 0$, we say that $A$ is monsingular. If $A$ is nonsingular, let $A^{\times}=\left\{u \in \omega:\left(u_{n} a_{n}\right) \in l_{1}, a \in A\right\}$. Clearly $A^{\times}$is sequence space. If $A$ is countable then $\lambda=A^{\times}$is countably echelon. If $\lambda$ is a sequence space, then $\lambda^{\times}$is called the Köthe dual of $\lambda$;

(f) certainly $\lambda \subseteq \lambda^{\times \times}: \lambda$ is perfect if $\lambda=\lambda^{\times \times}$.

Certainly both $\omega$ and $\phi$ are examples of normal perfect sequence spaces, and $\omega^{\times}=\phi, \phi^{\times}=\omega$. We now investigate some standard properties.

Proposition 2.2. (a) if $\lambda$ is perfect, it is normal;

(b) $A^{\times}$is perfect for any nonsingular set $A \subseteq \omega$;

(c) if $A, B$ are nonsingular and $A \subseteq B \subseteq \omega$, then $B^{\times} \subseteq A^{\times}$.

We shall introduce the following notion, which is a useful aid when proving the equivalence of topologies on tensor algebras.

Definition 2.3. The sequence space $\lambda$ is the diagonal transform of the sequence space $\mu$ if there exists $d \in \omega$ with $d_{n}>0$ for all $n \geqq 0$ such that $a=\left(a_{n}\right) \in \lambda$ if and only if $\left(a_{n} d_{n}\right) \in \mu$. We shall write this as $\lambda[d] \mu$. Clearly, if $\lambda[d] \mu$, then $\lambda^{\times}\left[d^{-1}\right] \mu^{\times}$.

If $\lambda$ is a sequence space then $\left(\lambda, \lambda^{\times}\right)$forms a dual pair with respect to the form

$$
(a, u)=\sum_{n \geqq 0} a_{n} u_{n} \quad a \in \lambda, u \in \lambda^{\times} .
$$


Thus we have the weak topology $\sigma\left(\lambda, \lambda^{\times}\right)$and the Mackey topology $\tau\left(\lambda, \lambda^{\times}\right)$on $\lambda$. Moreover, another natural topology may be defined on $\lambda$.

Definition 2.4. The normal topology $\tau\left(\lambda^{\times}\right)$on $\lambda$ is the Hausdorff locally convex topology on $\lambda$ given by the family of seminorms $\left\{p_{u}: u \in \lambda^{\times}\right\}$, where

$$
p_{u}(a)=\sum_{n \geqq 0}\left|u_{n} a_{n}\right| \quad a \in \lambda, u \in \lambda^{\times} .
$$

The principal result concerning these topologies is the following.

Proposition 2.5. $\sigma\left(\lambda, \lambda^{\times}\right) \leqq \tau\left(\lambda^{\times}\right) \leqq \tau\left(\lambda, \lambda^{\times}\right)$, so $\lambda^{\times}$is the topological dual of $\left(\lambda, \tau\left(\lambda^{\times}\right)\right)$.

\section{$\S 2 \cdot 2$. Order properties}

Let $\lambda$ be a normal sequence space. We can define a positive cone in $\lambda$ as follows:

$$
\lambda_{+}=\left\{a \in \lambda: a_{n} \geqq 0, n \geqq 0\right\} .
$$

Comparison with the definitions of Peressini [21] enables us to establish the following order properties of $\lambda_{+}$. The proofs are omitted.

Proposition 2.6. $\lambda_{+}$is generating, and $\lambda$ is an Archimedean vector lattice. If we equip $\lambda$ with the normal topology $\tau\left(\lambda^{\times}\right)$, then $\lambda_{+}$is a closed normal strict- $b$ cone for the topological vector lattice $\left(\lambda, \tau\left(\lambda^{\times}\right)\right)$.

In general, however, sequence spaces do not have order units.

Proposition 2.7. $\lambda$ possesses an order unit if and only if $\lambda$ is perfect and is a diagonal transform of $l_{\infty}$.

Proof. If $\lambda$ has an order unit $e$, for any $n \geqq 0$ find $\alpha(n)>0$ such that $\alpha(n) e-e(n) \in \lambda_{+}$whence $e_{n} \geqq \alpha(n)^{-1}>0$. Since $\lambda^{\times}=\left\{u \in \omega:\left(u_{n} e_{n}\right) \in l_{1}\right\}$, we see that $\lambda^{\times}[e] l_{1}$. Thus $\lambda^{\times \times}\left[e^{-1}\right] l_{\infty}$. If $a \in \lambda^{\times \times}$, then $\left(a_{n} / e_{n}\right) \in l_{\infty}$, whence $a \in \lambda$. Thus $\lambda=\lambda^{\times \times}$is perfect and $\lambda\left[e^{-1}\right] l_{\infty}$. The converse is trivial.

\section{$\S 2 \cdot 3$. Topological properties of $\left(\lambda, \tau\left(\lambda^{\times}\right)\right)$}

We shall now state various standard properties of the sequence space $\lambda$ when equipped with its normal topology.

Proposition $2 \cdot 8$. The completion of $\left(\lambda, \tau\left(\lambda^{\times}\right)\right)$is $\left(\lambda^{\times \times}, \tau\left(\lambda^{\times}\right)\right)$, so $\left(\lambda, \tau\left(\lambda^{* *}\right)\right)$ is complete if and only if $\lambda$ is perfect.

Proposition 2.9. $\left(\lambda, \tau\left(\lambda^{\times}\right)\right)$is Fréchet if and only if $\lambda$ is countably echelon. 
Proof. As observed in Köthe [17], if $\lambda=A^{\times}$is countably echelon, then $\tau\left(\lambda^{\times}\right)$may be defined by the countable family of seminorms $\left\{p_{a}: a \in A\right\}$. Conversely, if $\left(\lambda, \tau\left(\lambda^{\times}\right)\right)$is Fréchet, let $\left\{q_{k}: k \in N\right\}$ be a countable family of seminorms on $\lambda$ defining $\tau\left(\lambda^{\times}\right)$. For any $k \in N$ find $v(k) \in \lambda^{\times}$such that $q_{k} \leqq p_{v(k)}$. It is then clear that $A=\{v(k): k \in N\}$ is nonsingular and that $\lambda=A^{\times}$.

The condition for a sequence space $\left(\lambda, \tau\left(\lambda^{\times}\right)\right)$to be nuclear is wellknown, and was first established by Grothendieck [11] for perfect spaces. Proposition $50 \cdot 1$ of Trèves [27] extends the result.

Theorem 2.10. $\left(\lambda, \tau\left(\lambda^{\times}\right)\right)$is nuclear if and only if for every $u \in \lambda^{\times}$there exists $v \in \lambda^{\times}$and $\xi \in l_{1}$ such that $\left|u_{n}\right| \leqq\left|v_{n}\right||\xi|_{n}$ for all $n \geqq 0$.

It is always of interest to try to identify the strong topology on the dual of a locally convex space. In the case of a nuclear sequence space, we obtain the following.

Theorem 2.11. If $\lambda$ is perfect and $\left(\lambda, \tau\left(\lambda^{\times}\right)\right)$is nuclear, then the strong dual of $\left(\lambda, \tau\left(\lambda^{\times}\right)\right)$is $\left(\lambda^{\times}, \tau(\lambda)\right)$. Thus $\left(\lambda, \tau\left(\lambda^{\times}\right)\right)$is semireflexive.

Proof. Consider $\lambda^{\times}-l_{\infty}=\left\{a \in \omega:\left(a_{n} u_{n}\right) \in l_{\infty}, u \in \lambda^{\times}\right\}$equipped with the Hausdorff locally convex topology defined by the seminorms $p_{u}^{\infty}(a)=\sup _{n \geqq 0}\left|a_{n} u_{n}\right|$ for $a \in \lambda^{\times}-l_{\infty}, u \in \lambda^{\times}$. Clearly $\lambda \subseteq \lambda^{\times}-l_{\infty}$ and $p_{u}^{\infty}(a) \leqq p_{u}(a)$ for all $a \in \lambda, u \in \lambda^{\times}$. If $a \in \lambda^{\times}-l_{\infty}$ and $u \in \lambda^{\times}$, find $v \in \lambda^{\times}$and $\xi \in l_{1}$ such that $\left|u_{n}\right| \leqq\left|v_{n}\right|\left|\xi_{n}\right|$ for $n \geqq 0$. Then $\left(a_{n} u_{n}\right) \in l_{1}$ and $p_{u}(a) \leqq p_{v}^{\infty}(a)\|\xi\|_{1}$. Hence it follows that $\left(\lambda, \tau\left(\lambda^{\times}\right)\right) \equiv$ $\lambda^{\times}-l_{\infty}$. Hence (Garnir et al. [10] $\left.\mathrm{V} \cdot 1 \cdot 10\right) M$ is a bounded subset of $\left(\lambda, \tau\left(\lambda^{\times}\right)\right)$ if and only if there exists $a \in \lambda$ such that $\left|m_{n}\right| \leqq\left|a_{n}\right|$ for all $n \geqq 0$ and $m \in M$. The strong dual topology on $\lambda^{\times}$is the topology of uniform convergence on bounded subsets of $\left(\lambda, \tau\left(\lambda^{\times}\right)\right)$which (Köthe [17] 30.2.2) is the normal topology $\tau(\lambda)$. The last statement, which is immediate, should be compared with Proposition $4 \cdot 4 \cdot 11$ of Pietsch [22].

Corollary 2.12. If $\lambda$ is perfect and $\left(\lambda, \tau\left(\lambda^{\times}\right)\right)$is nuclear and dual nuclear, then $\left(\lambda, \tau\left(\lambda^{\times}\right)\right)$is reflexive.

\section{$\S 2 \cdot 4$. Convolution algebras}

We now proceed to try to assign an algebra structure to certain sequence spaces, by considering as a product law the natural convolution product. If $a, b \in \omega$ we look at $a * b \in \omega$, where

$$
(a * b)_{n}=\sum_{k=0}^{n} a_{k} b_{n-k} \quad n \geqq 0 .
$$


We wish to consider sequence spaces which are closed under this binary operation, which thus become commutative associative algebras with identity $e(0)$. It is of course well-known that $l_{1}$ is just such a sequence space, and when equipped with the normal topology $\tau\left(l_{\infty}\right)$-which is the same as the norm topology $-\left(l_{1}, \tau\left(l_{\infty}\right)\right)$ is a Banach algebra. Consequently, we make the following definition.

Definition 2-13. A sequence space $\lambda$ will be called $a$ convolution algebra if it is closed under the operation *.

We do not have a complete characterisation of convolution algebras. However, we do have the following partial result, which will completely characterise convolution algebras of type $h$ (see $\$ 2 \cdot 5$ below).

Proposition 2-14. If $\lambda$ is a normal sequence space such that $\left(n a_{n}\right) \in \lambda$ whenever $a \in \lambda$, then $\lambda$ is a convolution algebra if and only if for any $a, b \in \lambda$ there exists $c \in \lambda$ such that $\left|a_{m}\right|\left|b_{n}\right| \leqq\left|c_{m+n}\right|$ for all $m, n \geqq 0$.

Proof. If $\lambda$ is a convolution algebra and $a, b \in \lambda$, then setting $c=|a| *|b|$ suffices. Conversely, if $a, b \in \lambda$, choose $c \in \lambda$ as above. Then $\left|(a * b)_{n}\right| \leqq$ $\sum_{k=0}^{n}\left|a_{k}\right|\left|b_{n-k}\right| \leqq(n+1)\left|c_{n}\right|$ for all $n \geqq 0$, and hence $a * b \in \lambda$.

We do however have a complete characterisation of topological convolution algebras.

Theorem 2-15. Let $\lambda$ be a perfect sequence space. Then:

(a) $\left(\lambda, \tau\left(\lambda^{\times}\right)\right)$is a convolution algebra with separately continuous multiplication if and only if for all $a \in \lambda, u \in \lambda^{\times}$we have that $e(n) * a \in \lambda$ for all $n \geqq 0$, and $V(u, a) \in \lambda^{\times}$, where $V(u, a)_{n}=p_{u}(e(n) * a)$ for $n \geqq 0$;

(b) $\left(\lambda, \tau\left(\lambda^{\times}\right)\right)$is a convolution algebra with jointly continuous multiplication if and only if for each $u \in \lambda^{\times}$we can find $v, w \in \lambda^{\times}$such that $\left|u_{m+n}\right| \leqq\left|v_{m}\right|\left|w_{n}\right|$ for all $m, n \geqq 0$.

Proof. (a) if $\left(\lambda, \tau\left(\lambda^{\times}\right)\right)$is a separately continuous convolution algebra, for $a \in \lambda$ and $u \in \lambda^{\times}$we can find $v \in \lambda^{\times}$such that $p_{u}(a * b) \leqq p_{v}(b)$ for all $b \in \lambda$. Certainly $e(n) * a \in \lambda$ for all $n \geqq 0$ and $0 \leqq V(u, a)_{n} \leqq p_{v}(e(n))=\left|v_{n}\right|$, so $V(u, a) \in \lambda^{\times}$.

Conversely, if $a, b \in \lambda$ and $u \in \lambda^{\times}$, then

$$
\begin{aligned}
\sum_{n=0}^{N}\left|u_{n}\right|\left|(a * b)_{n}\right| & \left.\leqq \sum_{m=0}^{N} \sum_{n=0}^{N}\left|u_{m+n}\right|\left|a_{m}\right|\left|b_{n}\right|=\sum_{m=0}^{N} \sum_{n=0}^{N}\left|u_{m+n}\right| \mid(e(n)) * a\right)_{m+n}|| b_{n} \mid \\
& \leqq \sum_{n=0}^{N} V(u, a)_{n}\left|b_{n}\right| \leqq p_{V(u, a)}(b)
\end{aligned}
$$


for all $N \in \mathbb{N}$. Since this is true for all $u \in \lambda^{\times}$and $\lambda$ is perfect, it follows that $a * b \in \lambda$ and that $p_{u}(a * b) \leqq p_{V(u, a)}(b)$ for all $b \in \lambda$.

(b) if $\left(\lambda, \tau\left(\lambda^{\times}\right)\right)$is a jointly continuous convolution algebra, for any $u \in \lambda^{\times}$ find $v, w \in \lambda^{\times}$such that $p_{u}(a * b) \leqq p_{v}(a) p_{w}(b)$ for $a, b \in \lambda$. Since $e(m+n)=$ $e(m) * e(n)$ for $m, n \geqq 0$ it follows that $\left|u_{m+n}\right| \leqq\left|v_{m}\right|\left|w_{n}\right|$ for all $m, n \geqq 0$.

Conversely, if $a \in \lambda$ and $n \geqq 0$, for any $u \in \lambda^{\times}$choose $v, w^{*} \in \lambda^{\times}$as above. Then a simple calculation shows that $e(n) * a \in \lambda$ and $0 \leqq V(u, a)_{n} \leqq\left|u_{n}\right| p_{v}(a)$. Thus $\lambda$ is a convolution algebra and $p_{u}(a * b) \leqq p_{V(u, a)}(b) \leqq p_{v}(a) p_{w}(b)$ for all $b \in \lambda$.

The family of seminorms $\left\{p_{u}: u \in \lambda^{\times}\right\}$defining the topology $\tau\left(\lambda^{\times}\right)$on $\lambda$ is evidently very much larger than necessary, and consequently checking the conditions of Theorem $2 \cdot 15$ is made overcomplicated. We can, of course, simplify the problem as follows.

Definition 2.16. A subset $A$ of $\lambda^{\times}$is fundamental if, for every $u \in \lambda^{\times}$we can find $v \in A$ and $M>0$ such that $\left|u_{n}\right| \leqq M\left|v_{n}\right|$ for all $n \geqq 0$.

Clearly, if $A \subseteq \lambda^{\times}$is fundamental, then $A$ is nonsingular and $\left\{p_{u}: u \in A\right\}$ is a family of seminorms defining the topology $\tau\left(\lambda^{\times}\right)$on $\lambda$, while $A^{\times}=\lambda^{\times \times}$.

Recalling the definition of a locally multiplicatively convex algebra, we easily obtain the following results.

Theorem 2.17. (a) let $\lambda$ be a perfect sequence space and let $A \subseteq \lambda^{\times}$be fundamental. Then $\left(\lambda, \tau\left(\lambda^{\times}\right)\right)$is a jointly continuous convolution algebra if and only if for any $u \in A$ we can find $v, w \in A$ and $M \geqq 0$ such that $\left|u_{m+n}\right| \leqq$ $M\left|v_{m}\right|\left|w_{n}\right|$ for all $m, n \geqq 0$;

(b) let $\lambda$ be a perfect sequence space. Then $\left(\lambda, \tau\left(\lambda^{\times}\right)\right)$is a locally multiplicatively convex convolution algebra if and only if there exists a fundamental subset $A$ of $\lambda^{\times}$such that $\left|u_{m+n}\right| \leqq\left|u_{m}\right|\left|u_{n}\right|$ for all $m, n \geqq 0, u \in A$.

We consider now some of the standard sequence spaces (Peressini [21]):

(a) $\omega, \phi, l_{p}(0<p \leqq \infty), c, c_{o}$;

(b) $s=\left\{a \in \omega:\left(n^{k} a_{n}\right) \in c_{o}, k \in N\right\}, \quad s^{\prime}=\bigcup_{M \in N} \bigcup_{k \in N}\left\{a \in \omega:\left|a_{n}\right| \leqq M n^{k}, n \geqq 0\right\}$;

(c) $h=\left\{a \in \omega:\left(2^{n k} a_{n}\right) \in c_{o}, k \in \mathbb{N}\right\}, h^{\prime}=\bigcup_{z \in \in N} \bigcup_{k \in N}\left\{a \in \omega:\left|a_{n}\right| \leqq M 2^{n k}, n \geqq 0\right\}$;

(d) $h_{1}=\left\{a \in \omega:\left(2^{-n / k} a_{n}\right) \in l_{1}, k \in \mathbb{N}\right\}, h_{1}^{\prime}=\bigcup_{M \in N} \bigcup_{k \in N}\left\{a \in \omega:\left|a_{n}\right| \leqq M 2^{-n / k}, n \geqq 0\right\}$. The correspondence $a \leftrightarrow \sum_{n \geqq 0} a_{n} z^{n}$ provides a bijection between $h$ and the space $H(\boldsymbol{C})$ of entire functions, and a bijection between $h_{1}$ and the space of functions holomorphic on the open unit disc. All of these spaces, except $c$, are normal. 
We have the following listing of dual pairings $\left(\lambda, \lambda^{\times}\right):(\omega, \phi),(\phi, \omega),\left(l_{\infty}, l_{1}\right)$, $\left(l_{p}, l_{q}\right)$ for $1<p<\infty$ and $p^{-1}+q^{-1}=1,\left(l_{p}, l_{\infty}\right)$ for $0<p \leqq 1,\left(s, s^{\prime}\right),\left(s^{\prime}, s\right),\left(h, h^{\prime}\right)$, $\left(h^{\prime}, h\right),\left(h_{1}, h_{1}^{\prime}\right),\left(h_{1}^{\prime}, h_{1}\right)$.

If we define the sequence $\delta=(1,1,1, \cdots)$, then $\delta \in c \cap l_{\infty}$, but $(\delta * \delta)_{n}=n+1$, so $\delta * \delta \notin c \cup l_{\infty}$. For any $\alpha>0$ let $b^{\alpha}=\left((n+1)^{-\alpha}\right)$. Then $\left(b^{\alpha} * b^{\alpha}\right)_{n} \geqq(n+1)^{1-2 \alpha}$, and so $b^{1 / 2} \in c_{o}$ but $b^{1 / 2} * b^{1 / 2} \notin c_{o}$. For $1<p<\infty$ set $\alpha=\frac{1+p}{2 p}$. We can then show that $b^{\alpha} \in l_{p}$ but $b^{\alpha} * b^{\alpha} \notin l_{p}$. Thus we have shown that $c, c_{o}$ and $l_{p}(1<p \leqq$ $\infty)$ are not convolution algebras. However, all the other sequence spaces listed here are, and most of them are topological ones.

Theorem 2.18. (a) $(\phi, \tau(\omega))$ is a jointly continuous convolution algebra:

(b) equipped with their normal topologies, $\omega, l_{p}(0<p \leqq 1), s, h, h_{1}$ are locally multiplicatively convex convolution algebras:

(c) $s^{\prime}, h^{\prime}, h_{1}^{\prime}$ are convolution algebras.

Proof. (a) Alcantara [2], Schmüdgen [25];

(b) $\omega, l_{1}, s, h, h_{1}$ are countably echelon spaces $A^{\times}$with family $A=\{a(k)$ : $k \in N\}$ given by $a(k)=\sum_{r=0}^{k} e(r), a(k)=\delta, a(k)_{n}=(n+1)^{k}, a(k)_{n}=2^{k n}, a(k)_{n}=2^{-n / k}$ respectively. Since each of these families is submultiplicative as in Theorem $2 \cdot 17$ (b), the result follows. For $0<p<1$, if $a, b \in l_{p}$ then $a * b \in l_{p}$ and

$$
\|a * b\|_{p}^{p}=\sum_{n \geqq 0}\left|(a * b)_{n}\right|^{p} \leqq \sum_{n \geqq 0}\left[\sum_{k=0}^{n}\left|a_{k}\right|\left|b_{n-k}\right|\right]^{p} \leqq \sum_{n=0} \sum_{k \geqq 0}^{n}\left|a_{k}\right|^{p}\left|b_{n-k}\right|^{p} \leqq\|a\|_{p}^{p}|| b \|_{p}^{p} .
$$

Since $l_{p}^{\times}=l_{\infty},\left(l_{p}, \tau\left(l_{p}^{\times}\right)\right)$is a subalgebra of $\left(l_{1}, \tau\left(l_{1}^{\times}\right)\right)$.

(c) if $a, b \in s^{\prime}$ there exist $M, N, j, k \in \boldsymbol{N}$ with $\left|a_{n}\right| \leqq M(n+1)^{j},\left|b_{n}\right| \leqq$ $N(n+1)^{k}$ for $n \geqq 0$. Then $\left|(a * b)_{n}\right| \leqq M N(n+1)^{j+k+1}$ for $n \geqq 0$, so $a * b \in s^{\prime}$. The other proofs are similar.

Thus there are a reasonable number of topological convolution algebras. These will form the basis of our theory of tensor algebras. We finish this section with the following observation.

Remark 2.19. If $\left(\lambda, \tau\left(\lambda^{\times}\right)\right)$is a perfect locally multiplicatively convex convolution algebra, then $h \subseteq \lambda$, since Theorem $2 \cdot 17$ (b) implies that $\lambda^{\times} \subseteq h^{\prime}$.

\section{$\S 2 \cdot 5$. Sequence spaces of type $h$}

It will prove to be of interest later to restrict attention to a special class of sequence spaces. We introduce the following definition. 
Definition 2.20. A sequence space $\lambda$ is of type $h$ if $\left(2^{n} a_{n}\right) \in \lambda$ whenever $a \in \lambda$.

Clearly, $\phi, \omega, h$ are all of type $h$, and any diagonal transform of a type $h$ sequence space is also of type $h$. If $\lambda$ is of type $h$ then $\left(2^{n} u_{n} a_{n}\right) \in l_{1}$ for all $u \in \lambda^{\times}$ and $a \in \lambda$ (since $\left(2^{n} a_{n}\right) \in \lambda$ ), and hence $\left(2^{n} u_{n}\right) \in \lambda^{\times}$for all $u \in \lambda^{\times}$, so that $\lambda^{\times}$is also of type $h$. This last fact yields the following result.

Proposition 2.21. If $\lambda$ is of type $h$, then $\left(\lambda, \tau\left(\lambda^{\times}\right)\right)$is nuclear. If $\lambda$ is also perfect then $\left(\lambda, \tau\left(\lambda^{\times}\right)\right)$is nuclear, dual nuclear and reflexive.

Sequence spaces of type $h$ are in a particular sense fundamental to our theory. It is therefore gratifying to note that there is a large supply of such spaces. If $\lambda$ is any normal sequence space, for any $k \in N \cup\{0\}$ construct the following diagonal transform of $\lambda$,

$$
\lambda(k)=\left\{\left(2^{-k n} a_{n}\right): a \in \lambda\right\} .
$$

The normality of $\lambda$ implies that $\lambda=\lambda(0) \supseteq \lambda(1) \supseteq \cdots \supseteq \lambda(k) \supseteq \lambda(k+1) \supseteq \cdots \supseteq \phi$, and so we can define the new sequence space

$$
\lambda_{k}=\bigcap_{k \geqq 0} \lambda(k) .
$$

It is clear that if $\lambda$ is perfect or countably echelon, then so is $\lambda_{h}$.

Proposition 2.22. $\lambda_{h}$ is the largest sequence space of type $h$ contained in $\lambda$.

Proof. If $a \in \lambda_{h}$ then $a \in \lambda(k)$ for all $k \geqq 1$, so that $\left(2^{n} a_{n}\right) \in \lambda(k)$ for all $k \geqq 0$, so $\left(2^{n} a_{n}\right) \in \lambda_{h}$. Thus $\lambda_{h}$ is of type $h$. If $\mu$ is a sequence space of type $h$ contained in $\lambda$, then $\mu=\mu(k) \subseteq \lambda(k)$ for all $k \geqq 0$, so $\mu \subseteq \lambda_{k}$.

Of course, not all sequence spaces are of type $h$.

Corollary 2-23. If $h \subseteq \lambda \subseteq l_{\infty}$ then $\lambda_{h}=h$. Thus $s$ is not of type $h$.

Proof. $\quad\left(l_{\infty}\right)_{h}=h$, and so $s \neq h=s_{h}$.

In order to apply our subsequent theory, we need a good supply of jointly continuous convolution algebras which are of type $h$. It turns out that passage from $\lambda$ to $\lambda_{h}$ maintains algebraic properties in the Fréchet case.

Theorem 2-24. If $\lambda$ is a countably echelon space such that $\left(\lambda, \tau\left(\lambda^{\times}\right)\right)$ is a Fréchet topological (respectively locally multiplicatively convex) convolution algebra, then so is $\left(\lambda_{h}, \tau\left(\lambda_{k}\right)\right)$. 
Proof. Multiplication in $\left(\lambda, \tau\left(\lambda^{\times}\right)\right)$is jointly continuous. Now (Köthe [17] $30 \cdot 8 \cdot 1$ ) we have $\lambda_{h}^{\times}=\bigcup_{k \geqq 0} \lambda(k)^{\times}=\bigcup_{k \geqq 0}\left\{\left(2^{k n} u_{n}\right): u \in \lambda^{\times}\right\}$. Thus, if $A$ is a fundamental subset of $\lambda^{\times}$, then $\bigcup_{k \geq 0}\left\{\left(2^{k n} a_{n}\right): a \in A\right\}$ is a fundamental subset of $\lambda_{h}$. The result now follows from Theorem $2 \cdot 17$.

\section{$\S 3$. Tensor Algebras}

Let $E$ be a nuclear Fréchet space. A natural extension of the demands of quantum field theory leads us to study the so-called BU-algebra $E_{\otimes}=\bigoplus_{n \geqq 0} \bar{\otimes}^{n} E$ (Alcantara \& Dubin [4]), which was first introduced by Borchers [5] and Uhlmann [28]. For purposes of analysis it has proved useful to equip $E_{\otimes}$ with topologies other than the natural one (Alcantara [1], Hofmann [14], Wyss [31], Yngvason [32]). When equipped with these topologies, $E_{\otimes}$ is not complete. Relationships between these topologies are established in Hofmann [14]. In this paper we shall examine certain of these topologies distinguished by the fact that the completions of $E_{\otimes}$ are topological algebras.

\section{§3.1. General definitions}

Let $E$ be a complex nuclear Fréchet space equipped with the topology $t$. Then $t$ may be determined by a countable family $p=\left\{p_{r}: r \in \boldsymbol{N} \cup\{0\}\right\}$ of Hilbertian seminorms $p_{r}(x)=\langle x, x\rangle_{r}^{1 / 2}$ for $r \geqq 0, x \in E$, and without loss of generality we may assume that the family $p$ is directed, so that $p_{r} \leqq p_{r+1}$ for all $r \geqq 0$.

For any $n \in \boldsymbol{N}$, all the usual tensor product topologies coincide on $E_{n}=$ $\otimes^{n} E ; \varepsilon_{n}=\sigma_{n}=\pi_{n}=\iota_{n}$ (Jarchow [16]). When $n=1$ we have $\varepsilon_{1}=\sigma_{1}=\pi_{1}=\iota_{1}=t$. The completion of $E_{n}$ in this topology will be written $\hat{E}_{n}$. For $r_{1}, \cdots, r_{n} \geqq 0$ the map

$$
\langle x, y\rangle\rangle_{\left(r_{1}, \cdots, r_{n}\right)}^{(n)}=\sum_{i=1}^{k} \sum_{j=1}^{m}\left\langle x_{1}^{i}, y_{1}^{j}\right\rangle_{r_{1}} \cdots\left\langle x_{n}^{i}, y_{n}^{j}\right\rangle_{r_{n}},
$$

where $x=\sum_{i=1}^{k} x_{1}^{i} \otimes \cdots \otimes x_{n}^{i}, y=\sum_{j=1}^{m} y_{1}^{j} \otimes \cdots \otimes y_{n}^{j}$, is a preinner product on $E_{n}$. If we set $p_{\left(r_{1}, \cdots, r_{n}\right)}^{(n)}(x)=\left[\langle x, x\rangle_{\left(r_{1}, \cdots, r_{n}\right)}^{(n)}\right]^{1 / 2}$, then $\left\{p_{\left(r_{1}, \cdots, r_{n}\right)}^{(n)}: r, \cdots, r_{n} \geqq 0\right\}$ is a family of Hilbertian seminorms which defines the topology $\sigma_{n}$ on $E_{n}$. If we consider the family $\left\{p_{r}^{(n)}: r \geqq 0\right\}$ of diagonal seminorms given by $p_{r}^{(n)}=p_{(r, \cdots, r)}^{(n)}$, then since (Schmüdgen [25]) the $\sigma$-topology is monotonic, we have $p_{\left(r_{1}, \cdots, r_{n}\right)}^{(n)} \leqq p_{R}^{(n)}$, where $R=\max \left\{r_{1}, \cdots, r_{n}\right\}$, and so $\left\{p_{r}^{(n)}: r \geqq 0\right\}$ is a directed family of Hilbertian seminorms which determine the topology $\sigma_{n}$ on $E_{n}$. 
For notational completeness, the natural topology on $E_{0}=C$ may be determined by a trivial countable family of countable Hilbertian seminorms $\left\{p_{r}^{(0)}: r \geqq 0\right\}$ given by $p_{r}^{(0)}(\xi)=|\xi|$ for $r \geqq 0, \xi \in \mathbb{C}$. It is of interest to note that, for $j, k \geqq 0$, if $x \in \hat{E}_{j}$ and $y \in \hat{E}_{k}$, then $x \otimes y \in \hat{E}_{j+k}$ and $p_{r}^{(j+k)}(x \otimes y)=$ $p_{r}^{(j)}(x) p_{r}^{(k)}(y)$ for all $r \geqq 0$. This is called the cross-norm property. Clearly $\prod_{n \geqq 0} \hat{E}_{n}$ can be given the natural universal tensor algebra structure. It is of some interest to find subalgebras of $\prod_{n \geqq 0} \hat{E}_{n}$ bigger than $E_{\otimes}$, and to study topologies on these spaces which yield topological algebras. In particular we shall consider topologies similar to the $\tau_{\infty}$ topologies studied by Hofmann [14], which were originally introduced by Lassner [18].

Defimition 3.1. Let $\lambda$ be a normal sequence space. We define the vector space

$$
T(E, \lambda)=\left\{x=\left(x_{n}\right) \in \prod_{n \geqq 0} \hat{E}_{n}:\left(p_{r}^{(n)}\left(x_{n}\right)\right) \in \lambda, r \geqq 0\right\},
$$

and equip $T(E, \lambda)$ with the locally convex topology $t\left(\lambda^{\times}\right)$defined by the family $\left\{p_{u, r}: u \in \lambda^{\times}, r \geqq 0\right\}$ of seminorms, where

$$
p_{u, r}(x)=\sum_{n \geq 0}\left|u_{n}\right| p_{r}^{(n)}\left(x_{n}\right) \quad x \in T(E, \lambda) .
$$

Since $\phi \subseteq \lambda^{\times}$, the topology $t\left(\lambda^{x}\right)$ is Hausdorff. As observed by Hofmann [14], the space $T(E, \lambda)$ will in general depend on the choice of seminorms for $E$. It is clear that $T(E, \phi)=E_{\otimes}$ and $T(E, \omega)=\prod_{n \geq 0} \hat{E}_{n}$ are defined independently of this choice. In $\S 3.3$ we shall see that for sequence spaces of type $h, T(E, \lambda)$ is independent of this choice. Until then, when necessary we shall write $T(E, \lambda, p)$ and $t\left(\lambda^{\times}, p\right)$ to specify the particular family $p$ of seminorms.

However, $T(E, \lambda)$ is independent of the choice of $p$ to the following extent.

Lemma 3.2. (a) if $\lambda$ is a diagonal transform of $\mu$, then $\left(T(E, \lambda, p), t\left(\lambda^{\times}, p\right)\right)$ and $\left(T(E, \mu, p), t\left(\mu^{\times}, p\right)\right)$ are topologically isomorphic;

(b) $\left(T(E, \lambda), t\left(\lambda^{\times}\right)\right)$is, to within isomorphism, independent of a constant rescaling of the seminorms for $E$.

Proof. (a) if $\lambda[d] \mu$ then clearly the map $\left(x_{n}\right) \mapsto\left(d_{n} x_{n}\right)$ provides a linear isomorphism between $T(E, \lambda)$ and $T(E, \mu)$. Since $\lambda^{\times}\left[d^{-1}\right] \mu^{\times}$, this isomorphism is topological.

(b) if we replace $p=\left\{p_{r}: r \geqq 0\right\}$ by $q=\left\{q_{r}=K p_{r}: r \geqq 0\right\}$ for some $K>0$, then $q_{r}^{(n)}=K^{n} p_{r}^{(n)}$ for $r, n \geqq 0$, and hence $\left(T(E, \lambda, q), t\left(\lambda^{\times}, q\right)\right) \equiv\left(T(E, \mu, p), t\left(\mu^{\times}, p\right)\right)$ where $\lambda\left[\left(K^{-n}\right)\right] \mu$. Thus $\left(T(E, \lambda, p), t\left(\lambda^{\times}, p\right)\right) \equiv\left(T(E, \lambda, q), t\left(\lambda^{\times}, q\right)\right)$. 
Proposition 3.3. The topology $t(\phi)$ on $T(E, \omega)=\prod_{n \geq 0} \hat{E}_{n}$ is the product topology, and hence $(T(E, \omega), t(\phi))$ is complete.

Proof. The product topology on $T(E, \omega)$ is defined by the family of seminorms $\left\{P_{u, R}: u \in \phi, R \in \omega_{Z^{+}}\right\}$, where $P_{u, R}(x)=\sum_{n \geqq 0}\left|u_{n}\right| p_{R(n)}^{(n)}\left(x_{n}\right)$ for $x \in T(E, \omega)$ ( $\omega_{Z^{+}}$is the set of all $R \in \omega_{+}$such that $R(n) \in Z$ for all $n \geqq 0$ ).

If $u \in \phi$ and $r \geqq 0$, define $R \in \omega_{z^{+}}$by $R(n)=r$ for $n \geqq 0$. Then $p_{u, r}=P_{u, R}$. If $u \in \phi$ and $R \in \omega_{Z^{+}}$, then setting $\hat{r}=\max \left\{R(n): u_{n} \neq 0\right\}$ we see that $P_{u, R} \leqq p_{u, \hat{r}}$.

This result enables us to identify the completion of the space $(T(E, \lambda)$, $\left.t\left(\lambda^{\times}\right)\right)$. One difficulty that we have encountered in our analysis is that all the previous work on these topologies (Hofmann [14], [15], Wyss [31], Yngvason [32]) has been restricted to the space $T(E, \phi)=E_{\otimes}$ and hence sequences with only finitely many nonzero components. The following lemma bridges that gap.

Lemma 3.4. $T(E, \phi)$ is sequentially dense in $\left(T(E, \lambda), t\left(\lambda^{\times}\right)\right)$.

Proof. For any $n \in N$ consider $Q^{n}: T(E, \lambda) \rightarrow T(E, \phi)$ defined by $Q^{n} x=$ $\left(x_{0}, \cdots, x_{n}, 0,0, \cdots\right)$.

Then $p_{u, r}\left(x-Q^{n} x\right)=\sum_{k>n}\left|u_{k}\right| p_{r}^{(k)}\left(x_{k}\right) \rightarrow 0$ as $n \rightarrow \infty$ for all $u \in \lambda^{\times}, r \geqq 0$ and $x \in T(E, \lambda)$.

Let the completion of $\left(T(E, \lambda), t\left(\lambda^{\times}\right)\right)$be denoted $\hat{T}(E, \lambda)$. We have the following:

Theorem 3.5. $\hat{T}(E, \lambda)=\left(T\left(E, \lambda^{\times \times}\right), t\left(\lambda^{\times}\right)\right)$.

Proof. Firstly, since $\lambda^{x}$ is perfect, $t\left(\lambda^{x}\right)$ is the natural topology for $T\left(E, \lambda^{\times \times}\right)$. Let $\Phi(\lambda)$ be the $t\left(\lambda^{\times}\right)$-completion of $T(E, \phi)$. Then $\Phi(\lambda)$ is the $t\left(\lambda^{\times}\right)$-closure of $T(E, \phi)$ in $\hat{T}(E, \lambda)$, and so $\Phi(\lambda)=\hat{T}(E, \lambda)$ by Lemma 3.4. Now $t(\phi) \leqq t\left(\lambda^{\times}\right)$, and Theorem 6 (iii) of Yngvason [32] states that the $t(\phi)$-closure (in $T(E, \phi)$ ) of any graded subset of $T(E, \phi)$ is equal to its $t\left(\lambda^{\times}\right)$-closure, and hence $T(E, \phi)$ possesses a base of $t\left(\lambda^{\times}\right)$-neighbourhoods of zero which are $t(\phi)$ closed. Thus (Köthe [17] 18.5.4 (c)) we have that $\hat{T}(E, \lambda)=\Phi(\lambda) \subseteq \Phi(\omega)=$ $\hat{T}(E, \omega)=T(E, \omega)$, and hence $\hat{T}(E, \lambda)=\left\{x \in \prod_{n \geqq 0} \hat{E}_{n}: p_{u, r}(x)<\infty, u \in \lambda^{\times}, r \geqq 0\right\}=$ $\left\{x \in \prod_{n \geqq 0} \hat{E}_{n}:\left(p_{r}^{(n)}\left(x_{n}\right)\right) \in \lambda^{\times \times}, r \geqq 0\right\}=T\left(E, \lambda^{\times \times}\right)$.

\section{$\S 3 \cdot 2$. Tensor algebras}

We now proceed to consider sequence spaces $\lambda$ for which $T(E, \lambda)$ is a subalgebra of $T(E, \omega)$. The similarity between the algebra structure of $T(E, \omega)$ 
and the convolution product in sequence spaces now makes our earlier work vital, considered in conjunction with the cross-norm property. As usual, let $\lambda$ be a normal sequence space.

Theorem 3·6. If $\lambda$ is a convolution algebra, then $T(E, \lambda)$ is an algebra.

Proof. If $x, y \in T(E, \lambda)$ and $r \geqq 0$ define $X(r), Y(r) \in \lambda$ by setting $X(r)_{n}=$ $p_{r}^{(n)}\left(x_{n}\right), Y(r)_{n}=p_{r}^{(n)}\left(y_{n}\right)$. Then

$$
0 \leqq p_{r}^{(n)}\left((x y)_{n}\right) \leqq \sum_{k=0}^{n} p_{r}^{(n)}\left(x_{k} \otimes y_{n-k}\right)=\sum_{k=0}^{n} X(r)_{k} Y(r)_{n-k}=(X(r) * Y(r))_{n}
$$

for all $n \geqq 0$. Thus $\left(p_{r}^{(n)}\left((x y)_{n}\right)\right) \in \lambda$ for all $r \geqq 0$, so $x y \in T(E, \lambda)$.

We shall see that it is not necessary that $\lambda$ be a convolution algebra for $T(E, \lambda)$ to be an algebra. However, not all spaces $T(E, \lambda)$, where $\lambda$ is a normal sequence space, are algebras. For example, let $\left\{\Omega_{n}: n \geqq 0\right\}$ be the Hermite function Schauder basis for $\mathcal{S}(\mathbb{R})$, and let $p=\left\{p_{r}: r \geqq 0\right\}$ be the defining family of Hilbertian seminorms for $\mathcal{S}(\mathbb{R})$ given by $p_{r}(x)=\left\|N^{r} x\right\|_{2}(x \in \mathcal{S}(\mathbb{R}), r \geqq 0)$, where $N=\frac{1}{2}\left(x^{2}-\frac{d^{2}}{d x^{2}}+1\right)$ is the usual number operator, with $N \Omega_{n}=(n+1) \Omega_{n}$ for $n \geqq 0$. Consider $x \in T(\mathcal{S}(\mathbb{R}), \omega)$ defined by $x_{n}=\otimes^{n} \Omega_{0}(n \geqq 1), x_{0}=1$. Then $p_{r}^{(n)}\left(x_{n}\right)=1$ for all $r, n \geqq 0$, so that $x \in T\left(\mathcal{S}(\mathbb{R}), l_{\infty}\right)$. However $(x x)_{n}=(n+1) x_{n}$, so that $p_{r}^{(n)}\left((x x)_{n}\right)=n+1$ for all $r, n \geqq 0$, and hence $x x \notin T\left(\mathcal{S}(\mathbb{R}), l_{\infty}\right)$. Thus, with this choice of seminorms for $\mathcal{S}(\mathbb{R}), T\left(\mathcal{S}(\mathbb{R}), l_{\infty}\right)$ is not an algebra.

If we equip $\lambda$ with its normal topology, the topological properties of $\left(\lambda, \tau\left(\lambda^{\times}\right)\right)$are transferred over to $\left(T(E, \lambda), t\left(\lambda^{\times}\right)\right)$.

Proposition 3.7. If $A \subseteq \lambda^{\times}$is fundamental, then $\left\{p_{u, r}: u \in A, r \geqq 0\right\}$ is a family of seminorms defining the topology $t\left(\lambda^{\times}\right)$on $T(E, \lambda)$. In particular, if $\left(\lambda, \tau\left(\lambda^{\times}\right)\right)$is metrisable or complete, then so is $\left(T(E, \lambda), t\left(\lambda^{\times}\right)\right)$.

Theorem 3.8. Let $\lambda$ be a convolution algebra. If the product in $\left(\lambda, \tau\left(\lambda^{\times}\right)\right)$ is separately continuous (respectively jointly continuous, locally multiplicatively convex), then multiplication in $\left(T(E, \lambda), t\left(\lambda^{\times}\right)\right)$is separately continuous (respectively jointly continuous, locally multiplicatively convex).

Proof. If multiplication in $\lambda$ is separately continuous, for any $y \in T(E, \lambda)$, $u \in \lambda^{\times}$and $r \geqq 0$ find $v \in \lambda^{\times}$such that $p_{u}(a * Y(r)) \leqq p_{v}(a)$ for all $a \in \lambda$. Then we have

$$
p_{u, r}(x y) \leqq p_{u}(X(r) * Y(r)) \leqq p_{v}(X(r))=p_{v, r}(x)
$$

for all $x \in T(E, \lambda)$, and similarly $p_{u, r}(y x) \leqq p_{v, r}(x)$. 
If multiplication in $\lambda$ is jointly continuous, if $u \in \lambda^{\times}$find $v, w \in \lambda^{\times}$such that $\left|u_{m+n}\right| \leqq\left|v_{m}\right|\left|w_{n}\right|$ for $m, n \geqq 0$. Then

$$
p_{u, r}(x y) \leqq p_{u}(X(r) * Y(r)) \leqq p_{v}(X(r)) p_{w}(Y(r))=p_{v, r}(x) p_{w r}(y)
$$

for all $x, y \in T(E, \lambda)$.

The preceding paragraph and Theorem $2 \cdot 17$ establishes the last case.

\section{$\S 3 \cdot 3$. Nuclearity and sequence spaces of type $\boldsymbol{h}$}

Given any normal sequence space $\lambda$, a family of seminorms $p$ can be chosen such that $\left(T(E, \lambda, p), t\left(\lambda^{\times}, p\right)\right)$ is nuclear. Since the choice of $p$ is crucial, it is clear that, in order to discuss the nuclearity of our spaces $T(E, \lambda)$ sensibly, we are led to look for sequence spaces $\lambda$ such that $\left(T(E, \lambda), t\left(\lambda^{\times}\right)\right)$is independent of the choice of seminorms for $E$. Consequently this space will also be nuclear. We shall find that sequence space of type $h$ serve our purpose.

Work has already been done by Alcantara [1], Yngvason [32] and others in the case $\lambda=\phi$, and we shall use the techniques of Alcantara here.

Definition 3.9. A family of Hilbertian seminorms for $E$ is $\alpha$-directed if there exists $\alpha>1$ such that $p_{r+1} \geqq \alpha p_{r}$ for all $r \geqq 0$.

Of course, replacing the directed family $p=\left\{p_{r}: r \geqq 0\right\}$ by the equivalent family $p_{\alpha}=\left\{\alpha^{r} p_{r}: r \geqq 0\right\}$ if necessary, we see that $E$ can always be given an $\alpha$-directed family of seminorms.

Theorem 3-10. Let $p=\left\{p_{r}: r \geqq 0\right\}$ be a family of seminorms for $E$. Then $t\left(\lambda^{\times}, p\right)$ may be defined by the family $\left\{\hat{p}_{u, r}: u \in \lambda^{\times}, r \geqq 0\right\}$ of Hilbertian seminorms given by

$$
\hat{p}_{u, r}(x)=\left[\sum_{n \geqq 0}\left|u_{n}\right|^{2} p_{r}^{(n)}\left(x_{n}\right)^{2}\right]^{1 / 2} \quad x \in T(E, \lambda, p)
$$

if either $\left(\lambda, \tau\left(\lambda^{\times}\right)\right)$is nuclear or the family $p$ is $\alpha$-directed.

Proof. Clearly $\hat{p}_{u, r} \leqq p_{u, r}$ for $u \in \lambda^{\times}, r \geqq 0$. If $\left(\lambda, \tau\left(\lambda^{\times}\right)\right)$is nuclear, for any $u \in \lambda^{\times}$we can find $v \in \lambda^{\times}$and $\xi \in l_{2}$ such that $\left|u_{n}\right| \leqq\left|v_{n}\right|\left|\xi_{n}\right|$ for all $n \geqq 0$. Then $p_{u, r} \leqq\|\xi\|_{2} \hat{p}_{v, r}$ for all $r \geqq 0$.

Otherwise, if $p$ is $\alpha$-directed, for $r \geqq 0$ we have $p_{r} \leqq \alpha^{-1} p_{r+1}$, and so $p_{r}^{(n)} \leqq \alpha^{-n} p_{r+1}^{(n)}$ for $n \geqq 0$. Thus $p_{u, r} \leqq\left[\sum_{n \geqq 0} \alpha^{-2 n}\right]^{1 / 2} \hat{p}_{u, r+1}$ for all $u \in \lambda^{\times}, r \geqq 0$.

The results of Alcantara might lead us to hope that if $\left(\lambda, \tau\left(\lambda^{\times}\right)\right)$is nuclear, then so is $\left(T(E, \lambda, p), t\left(\lambda^{\times}, p\right)\right)$ for any choice of seminorms $p$. However this 
is not the case. To see this, equip $\mathcal{S}(\mathbb{R})$ with the family of seminorms $p$ defined by the number operator. The normal topology $\tau\left(s^{\prime}\right)$ is the usual nuclear Fréchet topology on $s$. If $\left(T(\mathcal{S}(\mathbb{R}), s, p), t\left(s^{\prime}, p\right)\right)$ were nuclear, for any $u \in s^{\prime}$ and $k \geqq 0$ we could find $v \in s^{\prime}$ and $m>k$ such that the canonical mapping $j: \mathscr{H}_{v, m} \rightarrow \mathscr{H}_{u, k}$ is nuclear, so Hilbert-Schmidt, where $\mathscr{H}_{u, k}$ is the Hilbert space completion of $T(S(\mathbb{R}), s, p) / \hat{p}_{u, k}^{-1}(0)$. We can also choose $m$ large enough so that the canonical mapping $\iota: \mathscr{H}_{m} \rightarrow \mathscr{H}_{k}$ is Hilbert-Schmidt, where $\mathscr{H}_{k}$ is the Hilbert space completion of $\mathcal{S}(\mathbb{R}) / p_{k}^{-1}(0)$.

Now $\left\{(n+1)^{-m} \Omega_{n}+p_{m}^{-1}(0): n \geqq 0\right\}$ is a countable orthonormal basis for $\mathcal{H}_{m}$ and so the Hilbert-Schmidt norm of $\iota$ is given by

$$
\|c\|_{2}=\left[\sum_{n \geqq 0} p_{k}\left((n+1)^{-m} \Omega_{n}\right)^{2}\right]^{1 / 2}=\left[\sum_{n \geqq 0}(n+1)^{-2(m-k)}\right]^{1 / 2}=\zeta(2 m-2 k)^{1 / 2}>1,
$$

where $\zeta$ is the Riemann zeta function. If we set $N(v)=\left\{t \geqq 0: v_{t} \neq 0\right\}$, then $\left\{E_{n_{1}, \cdots, n_{t}}+\hat{p}_{v, m}^{-1}(0): n_{1}, \cdots, n_{t} \geqq 0, t \in N(v)\right\}$ is an orthonormal basis for $\mathcal{H}_{v, m}$, where

$$
E_{n_{1}, \cdots, n_{t}}=\left(0, \cdots, 0, v_{t}^{-1}\left(n_{1}+1\right)^{-m} \cdots\left(n_{t}+1\right)^{-m} \Omega_{n_{1}} \otimes \cdots \otimes \Omega_{n t}, 0,0, \cdots\right) .
$$

Thus $\|j\|_{2}^{2}=\sum_{t \in N(v)} \sum_{i=1}^{t} \sum_{n_{i} \geqq 0} \hat{p}_{u, k}\left(E_{n_{1}, \cdots, n_{t}}\right)^{2}=\sum_{t \in N(v)}\left|u_{t} v_{t}^{-1}\right|^{2}\|c\|_{2}^{2 t}<\infty$, so we can choose $v \in s^{\prime}$ such that $v_{n} \geqq \| c||_{2}^{n}\left|u_{n}\right|$ for $n \geqq 0$. Hence, putting $u=\delta$ we see that $\left(\|c\|_{2}^{n}\right) \in s^{\prime}$, so we can find $M, k \in \mathbb{N}$ such that $\|\iota\|_{2}^{n} \leqq M(n+1)^{k}$ for $n \geqq 0$. This contradiction implies that $\left(T(\mathcal{S}(\mathbb{R}), s, p), t\left(s^{\prime}, p\right)\right)$ is not nuclear.

However, regardless of the nuclearity of $\lambda$ or otherwise, we do have the following result.

Theorem 3.11. If $p$ is an $\alpha$-directed family of seminorms for $E$, then $\left(T(E, \lambda, p), t\left(\lambda^{\times}, p\right)\right)$ is nuclear.

Proof. For $r \geqq 0$, choose $s>r$ such that the canonical mapping $\iota_{s r}: \mathscr{H}_{s} \rightarrow$ $\mathcal{H}_{r}$ is Hilbert-Schmidt $\left(\mathcal{H}_{r}\right.$ being the Hilbert space completion of $E / p_{r}^{-1}(0)$ ). For any $t>s$ the map $\iota_{t r}$ is Hilbert-Schmidt, and since $\iota_{t r}=\iota_{s r} \circ c_{t s}$ we have that $\left\|c_{t r}\right\|_{2} \leqq \sqrt{3}\left\|c_{t s}\right\|\left\|c_{s r}\right\|_{2} \leqq \sqrt{3} \alpha^{s-t}\left\|c_{s r}\right\|_{2}$ (Pietsch [22] $\left.\$ 2 \cdot 5\right)$, where $\left\|c_{t s}\right\|$ is the operator norm of $\iota_{t s}: \mathscr{H}_{t} \rightarrow \mathcal{H}_{s}$. Thus we can find $s>r$ such that $c_{s r}$ is HilbertSchmidt and $\left\|\iota_{s r}\right\|_{2}<1$.

For any $u \in \lambda^{\times}$, consider the canonical mapping $j_{s r}: \mathcal{H}_{u, s} \rightarrow \mathscr{H}_{u, r}$. An argument similar to the one above shows that $j_{s r}$ is Hilbert-Schmidt and that $\left\|j_{s r}\right\|_{2}$ is less than $\left[\sum_{t \in N(u)}\left\|c_{s r}\right\|_{2}^{2 t}\right]^{1 / 2}$. Since the composition of any two HilbertSchmidt operators is nuclear, it follows that $\left(T(E, \lambda, p), t\left(\lambda^{\times}, p\right)\right)$ is nuclear. 
One advantage of our proof over that of Alcantara is that we took a global change of seminorms to ensure that $\left\|\iota_{s r}\right\|_{2}<1$, whereas the change of seminorms used by Alcantara was local.

Theorem 3.12. If $\lambda$ is a normal sequence space of type $h$, then $(T(E, \lambda)$, $\left.t\left(\lambda^{\times}\right)\right)$is independent of the choice of seminorms for $E$, and hence is nuclear.

Proof. Let $p=\left\{p_{r}: r \geqq 0\right\}$ and $q=\left\{q_{s}: s \geqq 0\right\}$ be two equivalent directed families of Hilbertian seminorms for $E$. Let $x \in T(E, \lambda, p)$. For any $s \geqq 0$ we can find $r, k \geqq 0$ such that $q_{s} \leqq 2^{k} p_{r}$. Thus $q_{s}^{(n)} \leqq 2^{k n} p_{r}^{(n)}$ for $n \geqq 0$ and hence $\left(q_{s}^{(n)}\left(x_{n}\right)\right) \in \lambda$. Also, for any $u \in \lambda^{\times}$define $v=\left(2^{k n} u_{n}\right) \in \lambda^{\times}$. Then $q_{u, s}(x) \leqq p_{v, r}(x)$. The result now follows by symmetry.

The converse to this result is almost true.

Theorem 3.13. If $p$ is an $\alpha$-directed family of seminorms for $E$, and $\lambda$ is a normal sequence space, then $T(E, \lambda, p)=T\left(E, \lambda_{h}, p\right)$ algebraically, and $t\left(\lambda^{\times}, p\right) \leqq$ $t\left(\lambda_{h}^{\times}\right)$. If $\lambda$ is countably echelon, then $t\left(\lambda^{\times}, p\right)=t\left(\lambda_{h}^{\times}\right)$, and so $\left(T(E, \lambda, p), t\left(\lambda^{\times}, p\right)\right)$ and $\left(T\left(E, \lambda_{h}\right), t\left(\lambda_{h}^{\times}\right)\right)$are isomorphic.

Proof. Certainly $T\left(E, \lambda_{h}\right) \subseteq T(E, \lambda, p)$. If $x \in T(E, \lambda, p)$, for any $r, k \geqq 0$ we have $\left(p_{r}^{(n)}\left(x_{n}\right)\right),\left(p_{r+k}^{(n)}\left(x_{n}\right)\right) \in \lambda$. But $p_{r+k}^{(n)} \geqq \alpha^{k n} p_{r}^{(n)}$, so that $\left(\alpha^{k n} p_{r}^{(n)}\left(x_{n}\right)\right) \in \lambda$ for all $r, k \geqq 0$. Thus $\left(p_{r}^{(n)}\left(x_{n}\right)\right) \in \lambda_{h}$ for all $r \geqq 0$, so $x \in T\left(E, \lambda_{h}\right)$. Certainly $t\left(\lambda^{\times}, p\right) \leqq t\left(\lambda_{h}^{\times}\right)$, since $\lambda_{h} \subseteq \lambda$. If $\lambda$ is countably echelon, so is $\lambda_{h}$, and hence $t\left(\lambda^{\times}, p\right)$ and $t\left(\lambda_{h}^{\times}\right)$are two Fréchet topologies on $T\left(E, \lambda_{h}\right)$. Hence (Trèves [27] Theorem 17.1) it follows that $t\left(\lambda^{\times}, p\right)=t\left(\lambda_{h}^{\times}\right)$.

Corollary 3.14. If $\left(\lambda, \tau\left(\lambda^{\times}\right)\right)$is a Fréchet space such that $\left(T(E, \lambda), t\left(\lambda^{\times}\right)\right)$ is defined independently of the choice of seminorms for $E$, then without loss of generality we may assume that $\lambda=\lambda_{h}$ is a space of type $h$.

We notice that if $p$ is an $\alpha$-directed family of seminorms for $E$, then $T\left(E, c_{0}, p\right)=T\left(E,\left(c_{o}\right)_{h}\right)=T(E, h)$ is an algebra, even though $c_{0}$ itself is not a convolution algebra. Thus there is in general no two-way result about algebraic properties of $\lambda$ and those of $T(E, \lambda)$. However, if we restrict ourselves to sequence spaces of type $h$, then such a result does exist.

Theorem 3.15. If $\lambda$ is a perfect sequence space of type $h$, then $(T(E, \lambda)$, $\left.t\left(\lambda^{\times}\right)\right)$is Fréchet (respectively a separately continuous algebra, a jointly continuous algebra, locally multiplicatively convex) if and only if $\left(\lambda, \tau\left(\lambda^{\times}\right)\right)$is.

Proof. Choose $x \in E$ such that $p_{0}(x)=1$. The map $\xi: \omega \rightarrow T(E, \omega)$ given 
by $\xi(a)_{n}=a_{n} \otimes^{n} x$ is an algebra homomorphism which maps $\lambda$ to $T(E, \lambda)$, and whose restriction to $\lambda$ is $\left(\tau\left(\lambda^{\times}\right), t\left(\lambda^{\times}\right)\right)$-continuous. Also $p_{u}(a)=p_{u, 0}(\xi(a))$ for $a \in \lambda, u \in \lambda^{\times}$. Thus, if $\left\{q_{i}: i \in I\right\}$ is a family of seminorms defining $t\left(\lambda^{\times}\right)$, then $\left\{\tilde{q}_{i}: i \in I\right\}$ is a family defining $\tau\left(\lambda^{\times}\right)$, where $\tilde{q}_{i}(a)=q_{i}(\xi(a)$ ) (since the family $\left\{\tilde{p}_{u, r}: u \in \lambda^{\times}, r \geqq 0\right\}$ does).

If the seminorms $q_{i}$ are submultiplicative, then $q_{i}(\xi(a * b)) \leqq \tilde{q}_{i}(a) \tilde{q}_{i}(b)$ for $a, b \in \lambda, i \in I$, and so $a * b \in \lambda$ and $\tilde{q}_{i}(a * b) \leqq \tilde{q}_{i}(a) \tilde{q}_{i}(b)$ for $a, b \in \lambda, i \in I$. This establishes the final result. All the others are proved similarly.

We complete our preliminary discussion by making the following definition.

Definition 3.16. A regular tensor algebra is an algebra of the form $\left(T(E, \lambda), t\left(\lambda^{\times}\right)\right)$, where $\left(\lambda, \tau\left(\lambda^{\times}\right)\right)$is a sequence space of type $h$ which is a jointly continuous convolution algebra.

A regular tensor algebra is thus defined independently of the choice of seminorms for $E$, and is a nuclear jointly continuous topological algebra. Certainly $\phi, h, \omega$ all generate regular tensor algebras. If $\lambda$ is a countably echelon topological convolution algebra, then so is $\lambda_{h}$, and so $\lambda_{h}$ generates a Fréchet regular tensor algebra. Any Fréchet regular tensor algebra is nuclear Fréchet, and standard locally convex space theory (Pietsch [22], Schaefer [23], Trèves [27]) implies that it is Fréchet, barreled, bornological, Mackey, nuclear, reflexive and Montel, while its strong dual is barreled, bornological, complete, Mackey, nuclear, reflexive and Montel.

\section{$\S 4 . \quad$ Algebraic and Order Properties}

We shall now consider some of the properties of a regular tensor algebra. In $\S 4 \cdot 1$ we summarise the results found originally in Borchers [6] for $E_{\otimes}$, which extend simply to a more general tensor algebra. In later sections we introduce an involution to the algebra, and study the structure properties of the algebraic cone of positive elements. This cone has been extensively studied for $E_{\otimes}$ by Alcantara [1], [2], Hofmann [12], [13], [14], [15], Lassner [18], Lassner \& Uhlmann [20], Schmüdgen [25], Wyss [31] and Yngvason [32]. Extending these results to a more general regular tensor algebra can be done, but involves careful checking of the exact forms of sequences used. This is because, in the case $E_{\otimes}$, all sequences considered belong to $\phi^{\times}=\omega$, and so no care needs to be taken to control their behaviour. Now we need to know that all sequences belong to $\lambda^{x}$. 
Hereafter, let $\lambda$ be a perfect sequence space of type $h$ such that $\left(\lambda, \tau\left(\lambda^{\times}\right)\right)$is a (complete) convolution algebra with jointly continuous multiplication, and let $\left(T(E, \lambda), t\left(\lambda^{\times}\right)\right)$be an associated regular tensor algebra. It should be observed that most of these results can be proved more generally for a tensor algebra $\left(T(E, \lambda, p), t\left(\lambda^{\times}, p\right)\right.$ where $p$ is a family of seminorms for $E$ and $\lambda$ is just nuclear, and not of type $h$, but we shall for convenience assume the stronger condition.

\section{§ 4.1. Algebraic properties}

The results of this section may be summarised in the following Proposition.

Proposition 4.1. (a) $T(E, \lambda)$ has a multiplicative identity $e=(1,0,0, \cdots)$;

(b) $T(E, \lambda)$ has no zero divisors;

(c) the only idempotents of $T(E, \lambda)$ are 0 and $e$;

(d) the centre of $T(E, \lambda)$ is $C$.

Proof. (a), (b), (c): Borchers [6].

(d) if $y$ belongs to the centre of $T(E, \lambda)$, write $y=y_{0} e+z$, where $z$ belongs to the centre of $T(E, \lambda)$ and $z_{0}=0$. For any $x \in T(E, \lambda)$ we have that $(x z)_{2}=$ $(z x)_{2}$, so $x_{1} \otimes z_{1}=z_{1} \otimes x_{1}$ for all $x_{1} \in E$. For any $S \in E^{\prime}$, choose $0 \neq x_{1} \in E$ such that $S\left(x_{1}\right)=0$, and pick $T \in E^{\prime}$ such that $T\left(x_{1}\right) \neq 0$. Then $T\left(x_{1}\right) S\left(z_{1}\right)=$ $(T \otimes S)\left(x_{1} \otimes z_{1}\right)=(T \otimes S)\left(z_{1} \otimes x_{1}\right)=T\left(z_{1}\right) S\left(x_{1}\right)=0$, so that $S\left(z_{1}\right)=0$. Hence $z_{1}=0$. If $z_{0}=0, z_{1}=0, \cdots, z_{m-1}=0$, then $(x z)_{m+1}=(z x)_{m+1}$ for all $x \in T(E, \lambda)$, so $x_{1} \otimes z_{m}=$ $z_{m} \otimes x_{1}$ for all $x_{1} \in E$. For any $S_{1}, \cdots, S_{m} \in E^{\prime}$, find $0 \neq x_{1} \in E$ such that $S_{1}\left(x_{1}\right)=0$, and pick $T \in E^{\prime}$ such that $T\left(x_{1}\right) \neq 0$. Then $\left(S_{1} \otimes, \cdots, \otimes S_{m}\right)\left(z_{m}\right) T\left(x_{1}\right)=$ $\left(S_{1} \otimes \cdots \otimes S_{m} \otimes T\right)\left(z_{m} \otimes x_{1}\right)=0$ as above, so that $\left(S_{1} \otimes \cdots \otimes S_{m}\right)\left(z_{m}\right)=0$. Since $\otimes^{m} E^{\prime}$ is dense in $\hat{E}_{m}^{\prime}$, it follows that $z_{m}=0$. Hence, by induction, $z=0$, and so $y=y_{0} e \in \boldsymbol{C} e$.

\section{\$.2. Involutions and the algebraic cone}

Let us now suppose that $E$ possesses an involution * which is continuous, and let $p$ be a family of Hilbertian seminorms for $E$ which are *-invariant, so that $p_{r}\left(x^{*}\right)=p_{r}(x)$ for $r \geqq 0, x \in E$. This is equivalent to saying that $\left\langle x^{*}, y^{*}\right\rangle_{r}=$ $\overline{\langle x, y\rangle_{r}}$ for $r \geqq 0, x, y \in E$. Thus, if we extend $*$ to an involution on $E_{n}(n \in N)$ by defining $\left(x_{1} \otimes \cdots \otimes x_{n}\right)^{*}=x_{n}^{*} \otimes \cdots \otimes x_{1}^{*}$ and antilinearising, it is clear that $\left\langle x_{n}^{*}, y_{n}^{*}\right\rangle_{r}^{(n)}=\overline{\left\langle x_{n}, y_{n}\right\rangle_{r}^{(n)}}$ for $r \geqq 0, x_{n}, y_{n} \in E_{n}$, so that $p_{r}^{(n)}\left(x_{n}^{*}\right)=p_{r}^{(n)}\left(x_{n}\right)$ for $r \geqq 0$, $x_{n} \in E_{n}$. Hence each $\hat{E}_{n}$ has defined on it an involution which is invariant with respect to the seminorms $\left\{p_{r}^{(n)}: r \geqq 0\right\}$. Thus we may define an involution on 
$T(E, \lambda)$ by setting $\left(x^{*}\right)_{n}=x_{n}^{*}$ for $x=\left(x_{n}\right) \in T(E, \lambda)$, and it is now clear that $p_{u, r}\left(x^{*}\right)=p_{u, r}(x)$ for $u \in \lambda^{\times}, r \geqq 0, x \in T(E, \lambda)$. The following result is now evident.

Proposition 4.2. $\left(T(E, \lambda), t\left(\lambda^{\times}\right)\right)$is a topological $*_{\text {-algebra. }}$

We now consider the algebraic cone of positive elements for $T(E, \lambda)$ given by

$$
K(\lambda)=\left\{\sum_{i<\infty} a(i)^{*} a(i): a(i) \in T(E, \lambda)\right\}
$$

For technical reasons, we shall need to consider the subcone $K(\phi)$, defined similarly. Clearly $K(\phi) \subseteq K(\lambda)$. Since $T(E, \phi)$ is dense in $T(E, \lambda)$, the involution is continuous and multiplication is jointly continuous, it follows that $K(\phi)$ is $t\left(\lambda^{\times}\right)$-dense in $K(\lambda)$. Thus $\left.\overline{K(\phi)}{ }^{\lambda}=\overline{K(\lambda)}\right)^{\lambda}$, where $\bar{L}^{\lambda}$ denotes the $t\left(\lambda^{\times}\right)$-closure in $T(E, \lambda)$ of any subset $L$. Let us now consider the elementary properties of the cone $K(\lambda)$.

Proposition 4.3. $K(\lambda)$ is a proper strict-b cone in $\left(T(E, \lambda), t\left(\lambda^{\times}\right)\right)$.

Proof. That the cone is strict- $b$ follows from joint continuity of multipliaction. The proof of the propriety is a simple generalisation of the method of Borchers [6]. If $0 \neq y=\sum_{i<\infty} a(i)^{*} a(i) \in K(\lambda)$, let $\Lambda(i)$ be the index of the first nonzero component of $a(i)$. If we set $L=\min _{i} A(i)$, then the index of the first nonzero component of $y$ is $2 L$ and $y_{2 L}=\sum_{i<\infty} a(i)_{L}^{*} \otimes a(i)_{L}$, so that $\left(T^{*} \otimes T\right)\left(y_{2 L}\right)=$ $\sum_{i<\infty}\left|T\left(a(i)_{L}\right)\right|^{2}$ for any $T \in E_{L}^{\prime}$, where $T^{*} \in E_{L}^{\prime}$ is given by $T^{*}\left(x_{L}\right)=\overline{T\left(x_{L}^{*}\right)}$ for $x_{L} \in E_{L}$. Thus, if $0 \neq y \in K(\lambda) \cap(-K(\lambda))$, then the index of the first nonzero component of $-y$ is still $2 L$, and we see that $0=\left(T^{*} \otimes T\right)\left(y_{2 L}\right)=\sum_{i<\infty}\left|T\left(a(i)_{L}\right)\right|^{2}$ for all $T \in E_{L}^{\prime}$. Thus $T\left(a(i)_{L}\right)=0$ for all $T \in E_{L}^{\prime}$ and all $i$, so that $a(i)_{L}=0$ for all $i$. This contradicts the definition of $L$.

Corollary 4.4. If $\lambda$ is countably echelon, then $\left(T(E, \lambda), t\left(\lambda^{\times}\right)\right)$is an $I^{*}$ algebra in the sense of Alcantara \& Dubin [4].

\section{$\S 4 \cdot 3$. The closed cone $\overline{K(\lambda)}{ }^{\lambda}$}

Of course the closure $\overline{K(\lambda)}^{\lambda}$ of $K(\lambda)$ is a cone, but is it proper? The importance of this question lies in the fact (Peressini [21] Proposition 1 19) that $\overline{K(\lambda)}{ }^{\lambda}$ is proper if and only if the continuous positive linear functionals $K(\lambda)^{\prime}$ separate points in $T(E, \lambda)$, where 


$$
K(\lambda)^{\prime}=\left\{T \in T(E, \lambda)^{\prime}: T(x) \geqq 0, x \in K(\lambda)\right\},
$$

and so we have an adequate supply of continuous positive linear functionals. Before we start our analysis of this problem, we need the following characterisation of the dual $T(E, \lambda)^{\prime}$ of $\left(T(E, \lambda), t\left(\lambda^{\times}\right)\right)$.

Theorem 4.5. If $S: T(E, \lambda) \rightarrow C$ is linear, then $S \in T(E, \lambda)^{\prime}$ if and only if we can find a sequence $\left(S_{n}\right), r \geqq 0$ and $u \in \lambda^{\times}$such that:

(a) $S_{n} \in \hat{E}_{n}^{\prime}$ for all $n \geqq 0$;

(b) $\left|S_{n}\left(x_{n}\right)\right| \leqq\left|u_{n}\right| p_{r}^{(n)}\left(x_{n}\right)$ for all $n \geqq 0$ and $x_{n} \in \hat{E}_{n}$;

(c) $S(x)=\sum_{n \geqq 0} S_{n}\left(x_{n}\right)$ for all $x=\left(x_{n}\right) \in T(E, \lambda)$.

Proof. If $S \in T(E, \lambda)^{\prime}$ we can find $r \geqq 0$ and $u \in \lambda^{\times}$such that $|S(x)| \leqq$ $p_{u, r}(x)$ for all $x \in T(E, \lambda)$. For any $n \geqq 0$ define the linear map $S_{n}: \hat{E}_{n} \rightarrow C$ by setting $S_{n}\left(x_{n}\right)=S\left(x_{n}^{\sim}\right)$, where $\sim: \hat{E_{n}} \rightarrow T(E, \lambda)$ is the natural injection. Clearly $\left|S_{n}\left(x_{n}\right)\right| \leqq p_{n, r}\left(x_{n}\right)=\left|u_{n}\right| p_{r}^{(n)}\left(x_{n}\right)$ for $n \geqq 0$ and $x_{n} \in \hat{E}_{n}$, so certainly $S_{n} \in \hat{E}_{n}^{\prime}$. Finally $S(x)-\sum_{n=0}^{N} S_{n}\left(x_{n}\right)=S\left(x-Q^{N} x\right)$ for any $N \geqq 0$ and $x \in T(E, \lambda)$, so the desired result is obtained by letting $N \rightarrow \infty$.

Conversely, it is clear that if $\left(S_{n}\right), r \geqq 0$ and $u \in \lambda^{\times}$satisfy (a) and (b), then (c) defines $S \in T(E, \lambda)^{\prime}$ such that $|S(x)| \leqq p_{u, r}(x)$ for all $x \in T(E, \lambda)$.

Now Alcantara [1], Hofmann [12] and Yngvason [32] have all shown that $K(\phi)$ is a normal cone in $(T(E, \phi), t(\omega))$. Hence $\overline{K(\phi)}^{\phi}$ is also normal, hence proper. This result is not true for all convolution algebras $\lambda$ of type $h$. Indeed, a simple application of the Cauchy-Schwarz inequality shows that the only elements of $K(\omega)^{\prime}$ are positive multiples of the functional $N$, where $N_{0}=1$ and $N_{n}=0$ for $n \geqq 1$.

To begin with, we notice that some convolution algebras $\lambda$ possess nontrivial multiplicative functionals. If $S \in E^{\prime}$ we can find $r \geqq 0, J>0$ such that $|S(x)| \leqq J p_{r}(x)$ for $x \in E$. For $n \in \mathbb{N}$ we have $\otimes^{n} S \in \hat{E}_{n}^{\prime}$ and $\left|\left(\otimes^{n} S\right)\left(x_{n}\right)\right| \leqq$ $J^{n}\left(p_{r} \otimes_{\varepsilon} \cdots \bigotimes_{\varepsilon} p_{r}\right)\left(x_{n}\right) \leqq J^{n} p_{r}^{(n)}\left(x_{n}\right)$ for $x_{n} \in \hat{E}_{n}$. Thus the sequence $\left(\otimes^{n} S\right)$ defines an element $M_{S}$ in $T(E, \lambda)^{\prime}$ provided that $\left(J^{n}\right) \in \lambda^{\times}$, which is certainly true if $\lambda \subseteq h$. The map $M_{S}$ is multiplicative and positive if $S=S^{*}$; it is clearly the same as the map $\left.\frac{1}{1-S}\right|_{\times}$of Borchers [6]. Thus if $\lambda \subseteq h$ wo have a large supply of nontrivial positive linear functionals on $T(E, \lambda)$. Indeed, a conversation with Professor K. Schmüdgen led us to consider the following argument using a generalization of the left regular representation.

Theorem 4.6. If $\lambda \subseteq h$, then $\overline{K(\lambda)}$ is proper. 
Proof. For any $x \in E$ we consider $\pi(x): T(E, \lambda) \rightarrow T(E, \lambda)$ given by $\pi(x) y=\tilde{x} y$ for $y \in T(E, \lambda)$. Fix $r \geqq 0$. If $y \in T(E, \lambda)$ we have $\hat{p}_{\delta, r}[\pi(x) y]^{2}=$ $p_{r}(x)^{2} \hat{p}_{\delta, r}(y)^{2}$, so we may define a continuous linear map $\hat{\pi}_{r}: E \rightarrow \mathscr{B}\left(\mathcal{H}_{\delta, r}\right)$ such that $\left\|\hat{\pi}_{r}(x)\right\|=p_{r}(x)$ for $x \in E$. Define $\sigma_{r}: E \rightarrow \mathscr{B}\left(\mathcal{H}_{\delta, r}\right)$ by setting $\sigma_{r}(x)=$ $\hat{\pi}_{r}(x)+\hat{\pi}_{r}\left(x^{*}\right)^{*}$. Then $\sigma_{r}$ is linear, $\left\|\sigma_{r}(x)\right\| \leqq 2 p_{r}(x)$ and $\sigma_{r}\left(x^{*}\right)=\sigma_{r}(x)^{*}$ for all $x \in E$. Let $\Sigma_{r}$ be the *-algebra representation of $E_{\otimes}=\bigoplus_{n \geq 0} \bar{\otimes}^{n} E$ defined by $\sigma_{r}$ (and the requirement that $\Sigma_{r}(e)=I$ ).

If $x_{n}=\sum_{i} x_{1}^{i} \otimes \cdots \otimes x_{n}^{i} \in \otimes^{n} E$ then $\hat{p}_{\delta, r}\left[\Sigma_{r}\left(x_{n}\right) \xi\right] \leqq \sum_{i} \hat{p}_{\delta, r}\left[\sigma_{r}\left(x_{1}^{i}\right) \cdots \sigma_{r}\left(x_{n}^{i}\right) \xi\right] \leqq$ $2^{n} \sum_{i} p_{r}\left(x_{1}^{i}\right) \cdots p_{r}\left(x_{n}^{i}\right) \hat{p}_{\delta, r}(\xi)$ for all $\xi \in \mathcal{H}_{\delta, r}$. Thus $\hat{p}_{\delta, r}\left[\Sigma_{r}\left(x_{n}\right) \xi\right] \leqq 2^{n}\left(\bigotimes_{\pi}^{n} p_{r}\right)\left(x_{n}\right) \times$ $\hat{p}_{\delta, r}(\xi)$ for all $x_{n} \in E_{n}$ and $\xi \in \mathcal{H}_{\delta, r}$. A simple generalization of Proposition 7.3.2 of Pietsch [22] shows that there exists $K>0, s \geqq 0$ (depending on $r$ only) such that $\bigotimes_{\pi}^{n} p_{r} \leqq K^{n} p_{s}^{(n)}$ for all $n \geqq 0$. If we define $v=\left((2 K)^{n}\right) \in h^{\prime} \subseteq \lambda^{\times}$, it is clear that $\hat{p}_{\delta, r}\left[\Sigma_{r}(x) \xi\right] \leqq p_{v, s}(x) \hat{p}_{\delta, r}(\xi)$ for all $x \in E_{\otimes}$ and $\xi \in \mathcal{H}_{\delta, r}$. Since $E_{\otimes}$ is dense in $T(E, \lambda)$, it follows that $\Sigma_{r}$ extends to a continuous *-representation $\hat{\Sigma}_{r}$ of $T(E, \lambda)$.

For any $0 \leqq \mu \leqq 1$ it is clear that the map $x \mapsto x(\mu)$ is a continuous $*_{\text {-endo }}$ morphism of $T(E, \lambda)$, where $x(\mu)=\left(\mu^{n} \cdot x_{n}\right)$. Thus if $x \in \overline{K(\lambda)}^{\lambda}$ then $x(\mu) \in \overline{K(\lambda)}{ }^{\lambda}$ for all $0 \leqq \mu \leqq 1$.

If $x \in \overline{K(\lambda)} \bar{C}^{\lambda} \cap(-\overline{K(\lambda)})$, for any $r \geqq 0$ and $\xi \in \mathcal{H}_{\delta, r}$ the map $y \mapsto\left\langle\xi, \hat{\Sigma}_{r}(y) \xi\right\rangle$ belongs to $K(\lambda)^{\prime}$, and hence $\left\langle\xi, \hat{\Sigma}_{r}(x(\mu)) \xi\right\rangle=0$ for all $0 \leqq \mu \leqq 1$. Since $\hat{\Sigma}_{r}(x(\mu))$ is self-adjoint, a standard argument shows that $\sum_{n \geq 0} \mu^{n} \hat{\Sigma}_{r}\left(x_{n}\right) \xi=\hat{\Sigma}_{r}(x(\mu)) \xi=0$ for $0 \leqq \mu \leqq 1$. Thus $\hat{\Sigma}_{r}\left(x_{n}\right) \xi=0$ for all $n \geqq 0$, and so $\hat{p}_{\delta, r}\left[\hat{\Sigma}_{r}\left(x_{n}\right) e\right]=0$ for all $r, n \geqq 0$. Looking at the $n^{\text {th }}$ term of this sum, we see that $p_{r}^{(n)}\left(x_{n}\right)=0$ for all $r, n \geqq 0$, so $x=0$.

Thus we can find a large number of regular tensor algebras with proper closed cone, and $\left(T(E, h), t\left(h^{\prime}\right)\right)$ is a locally multiplicatively convex regular tensor algebra with closed cone. Indeed $T(E, h)$ is the only such algebra, because it turns out that the reverse implication in Theorem 4.6 is true. We shall prove this in two stages, starting with the simple case $E=C$. Clearly then $\left(T(E, \lambda), t\left(\lambda^{\times}\right)\right)=\left(\lambda, \tau\left(\lambda^{\times}\right)\right)$, and

$$
\begin{aligned}
& K(\lambda) \leftrightarrow k(\lambda)=\left\{\sum_{i<\infty} \overline{a(i)} * a(i): a(i) \in \lambda\right\}, \\
& K(\lambda)^{\prime} \leftrightarrow k(\lambda)^{\prime}=\left\{u \in \lambda^{\times}:(u, \bar{a} * a) \geqq 0, a \in \lambda\right\},
\end{aligned}
$$

so that $k(\lambda)^{\prime}$ is precisely the collection of sequences in $\lambda^{\times}$of positive type. Thus, if $u \in k(\lambda)^{\prime}$ we can find a positive finite measure $\mu$ on $\boldsymbol{R}$ such that $u_{n}=\int_{\mathbb{R}} t^{n} d \mu(t)$ for $n \geqq 0$. If $\mu(\mathbb{R}-\{0\})=0$ it is clear that $u=\mu(\mathbb{R}) e(0)$. 
Otherwise we can find $\varepsilon>0$ such that $M_{\varepsilon}=\mu(\{t:|t| \geqq \varepsilon\})>0$, and then $u_{2 n} \geqq$ $\varepsilon^{2 n} M_{\varepsilon}$ for all $n \geqq 0$, so that $\left(1,0, \varepsilon^{2}, 0, \varepsilon^{4}, \cdots\right)$ belongs to $\lambda^{\times}$. Since $\lambda$ is of type $h,\left\{u \in \omega:\left(u_{2 n}\right) \in h^{\prime},\left(u_{2 n+1}\right)=0\right\} \subseteq \lambda^{\lambda}$. If $a \in \lambda$ then $\left(a_{2 n} u_{n}\right) \in l_{1}$ for all $u \in h^{\prime}$, so that $\left(a_{2 n}\right) \in h$. But also $a * e(1) \in \lambda$ and so $\left(a_{2 n+1}\right) \in h$. Thus $a \in h$. We have proved the following result.

Proposition 4.7. Either $\lambda \subseteq h$ or $k(\lambda)^{\prime}=\{\alpha e(0): \alpha \geqq 0\}$.

Corollary 4.8. $\overline{k(\lambda)}{ }^{\lambda}$ is proper if and only if $\lambda \subseteq h$.

To extend this result to the general case, we need some more technical equipment. For any $s \in \boldsymbol{N}$ define

$$
\lambda_{s}=\left\{a \in \omega: a=\left(b_{s n}\right)_{n} \text { for some } b \in \lambda\right\} .
$$

Clearly $\lambda_{s}$ is a sequence space, and we notice that $\lambda_{1}=\lambda$. The following results are easily proved.

Lemma 4.9. (a) $\left(\lambda_{s}, \tau\left(\lambda_{s}^{\times}\right)\right)$is a complete convolution algebra with jointly continuous multiplication;

(b) $\left(\lambda_{s}\right)^{\times}=\left(\lambda^{\times}\right)_{s}$;

(c) $\lambda_{s}$ is of type $h$, and $\lambda_{s} \subseteq h$ if and only if $\lambda \subseteq h$.

We also introduce the following notation. If $a \in \lambda_{s}$, let $a[s] \in \lambda$ be given by setting $a[s]_{n}=a_{n / s}$ if $s$ divides $n$, and $a[s]_{n}=0$ otherwise.

Theorem 4.10. Either $\lambda \subseteq h$ or $K(\lambda)^{\prime}=\{\alpha N: \alpha \geqq 0\}$.

Proof. Suppose that $\lambda$ is not contained in $h$, and pick $T \in K(\lambda)^{\prime}$. Fix $s \in \boldsymbol{N}$, and choose $x=x^{*} \in \hat{E}_{s}$. For any $a \in \lambda_{s}$ consider $x(a) \in T(E, \omega)$ given by $x(a)_{s n}=a_{n} \otimes^{n} x$ for $n \geqq 0$, and $x(a)_{n}=0$ if $s$ does not divide $n$. Clearly $p_{r}^{(n)}\left(x(a)_{n}\right)$ $=\left|a[s]_{n}\right| p_{r}^{(s)}(x)^{n / s}$ for all $r, n \geqq 0$, so that $x(a) \in T(E, \lambda)$. Now the map $a \mapsto x(a)$ is a *-algebra homomorphism from $\lambda_{s}$ to $T(E, \lambda)$. For any $u \in \lambda^{\times}$and $r \geqq 0$, if we define $v=\left(p_{r}^{(s)}(x)^{n} u_{s n}\right)_{n} \in\left(\lambda_{s}\right)^{\times}$, it is clear that $p_{u, r}(x(a))=p_{v}(a)$ for all $a \in \lambda_{s}$, and so the homomorphism is continuous from $\left(\lambda_{s}, \tau\left(\lambda_{s}^{\times}\right)\right)$to $\left(T(E, \lambda), t\left(\lambda^{\times}\right)\right)$. Thus the map $T_{x}: a \mapsto T(x(a))$ belongs to $k\left(\lambda_{s}\right)^{\prime}$. Now $\lambda_{s}$ is not contained in $h$, so $T_{x}=\beta e(0)$ for some $\beta \geqq 0$. In particular $0=T_{x}(e(1))=T(x(e(1)))=T_{s}(x)$. Thus $T_{s}(x)=0$ for all $x=x^{*} \in \hat{E}_{s}$, and hence $T_{s}=0$. This is true for all $s \in N$, so $T=\alpha N$ for some $\alpha \geqq 0$.

Corollary 4.11. $\overline{K(\lambda)}^{\lambda}$ is proper if and only if $\lambda \subseteq h$. 


\section{$\S$ 4. A. Criteria for mormality}

There are few simple results concerning normality of the cone $K(\lambda)$ in $\left(T(E, \lambda), t\left(\lambda^{\times}\right)\right)$, and those which can be tested are highly restrictive, like those of Hofmann [12]. We start with a few general observations. For any $T \in K(\lambda)^{\prime}$ the map

$$
p_{T}(x)=T\left(x^{*} x\right)^{1 / 2} \quad x \in T(E, \lambda)
$$

is a continuous seminorm on $\left(T(E, \lambda), t\left(\lambda^{\times}\right)\right.$) (Lassner [19]). The topology defined by the family of seminorms $\left\{p_{T}: T \in K(\lambda)^{\prime}\right\}$ is called the state topology, written $t_{S}$. Clearly $t_{S}$ is Hausdorff if and only if $\overline{K(\lambda)}^{\lambda}$ is proper. We say that the states determine the topology if $t_{S}=t\left(\lambda^{\times}\right)$. Since $\left(T(E, \lambda), t\left(\lambda^{\wedge}\right)\right)$ is nuclear, results of Alcantara [1] and Yngvason [32] yield the following result.

Proposition $4 \cdot 12 . K(\lambda)$ is $t\left(\lambda^{\times}\right)$-normal if and only if the states determine the topology:

It should be noted that there exist no locally multiplicatively convex regular tensor algebras with normal cones. To see this, if $\left(T(E, \lambda), t\left(\lambda^{\times}\right)\right)$is a complete locally multiplicatively convex regular tensor algebra, for any $T \in K(\lambda)^{\prime}$ Brooks [7] has shown that the Gelfand-Naimark-Segal representation $\left(\pi_{T}, \mathcal{H}_{T}, \Omega_{T}\right)$ of $T(E, \lambda)$ is a continuous representation of $T(E, \lambda)$ on $\mathcal{B}\left(\mathcal{H}_{T}\right)$. Thus the function $q_{T}(x)=\left\|\pi_{T}(x)\right\|(x \in T(E, \lambda))$ is a continuous $C^{*}$-seminorm on $T(E, \lambda)$ such that $p_{T} \leqq q_{T}$, provided that $T(e)=1$. Thus the collection of $C^{*}$-seminorms $\left\{q_{T}: T \in K(\lambda)^{\prime}\right\}$ defines a topology $t_{G N S}$ on $T(E, \lambda)$ such that $t_{S} \leqq t_{G N S} \leqq t\left(\lambda^{\times}\right)$. If $K(\lambda)$ were also $t\left(\lambda^{\times}\right)$-normal, then $t\left(\lambda^{\times}\right)=t_{G N S}$, and so $T(E, \lambda)$ would be a $b^{*}$-algebra, and so symmetric. As observed in the previous section, the only serious candidate for yielding a locally multiplicatively convex regular tensor algebra with normal cone is $h$, and certainly $T(E, h)$ is not symmetric in general. To see this, choose $0 \neq x=x^{*}$ in $E$ and let $y=\left(\frac{1}{n !} \otimes^{n} x\right) \in T(E, h)$. Then $y^{*} y=\left(\frac{1}{n !} 2^{n} \otimes^{n} x\right)$. If $T(E, h)$ were symmetric, then $M_{S}\left(e+y^{*} y\right) \neq 0$ for all $S \in E^{\prime}$. But since $M_{S}\left(e+y^{*} y\right)=1+\exp [2 S(x)]$ and we can find $S \in E^{\prime}$ such that $S(x)=\frac{1}{2} i \pi$, we obtain the required contradiction.

A variety of conditions for normality of the cone can be found by generalizing the arguments of Lassner \& Uhlmann [20], Hofmann [12] and Schmüdgen [25]. These criteria demand that $\lambda$ be (essentially) very "close" to $\phi$, since $\lambda^{x}$ must contain a large number of sequences which diverge to infinity very 
rapidly. To be able to apply the techniques of the above authors, we notice that since $\overline{K(\phi)} \bar{K}^{\lambda}=\overline{K(\lambda)}^{\lambda}$, the normality of $K(\lambda)$ in $\left(T(E, \lambda), t\left(\lambda^{\times}\right)\right)$is equivalent to the normality of $K(\phi)$ (Peressini [21] Proposition $1 \cdot 5$, Corollary $1 \cdot 6$ ).

As in Lassner \& Uhlmann [20], Hofmann [12], if $g=\sum_{i<\infty} a(i)^{*} a(i) \in K(\phi)$, for any $r, n \geqq 0$ define the number

$$
L_{r}^{n}(g)=\left[p_{r}^{(2 n)}\left[\sum_{i<\infty} a(i)_{n}^{*} \otimes a(i)_{n}\right]\right]^{1 / 2} .
$$

Clearly the components $L_{r}^{n}(g)$ depend on the representation of $g$, but this turns out to be unimportant. The fundamental properties of the components $L_{r}^{n}(g)$ are as follows:

$$
\begin{aligned}
& p_{r}^{(n)}\left(g_{n}\right) \leqq \sum_{k=0}^{n} L_{r}^{k}(g) L_{r}^{n-k}(g), \\
& p_{r}^{(2 n)}\left(g_{2 n}\right) \geqq L_{r}^{n}(g)^{2}-2 \sum_{k=1}^{n} L_{r}^{n+k}(g) L_{r}^{n-k}(g),
\end{aligned}
$$

and $L_{r}^{n}\left(g_{1}+g_{2}\right) \geqq L_{r}^{n}\left(g_{1}\right)$ for all $r, n 0, g, g_{1}, g_{2} \in K(\phi)$.

We now introduce various types of sequences. If $a \in \omega$, define the infinite symmetric matrices $H(a)=\left\|H(a)_{i j}\right\|_{i, j \geqq 0}$ and $\Delta(a)=\left\|\Delta(a)_{i j}\right\|_{i, j \geqq 0}$ by setting

$$
H(a)_{i j}=\left\{\begin{array}{cl}
a_{n} & i=j=n \\
-a_{n} & i \neq j, i+j=2 n \\
0 & \text { otherwise, }
\end{array}\right.
$$

and

$$
\Delta(a)_{i j}=\left|a_{i}\right|^{2} \delta_{i j}
$$

Definition 4-13. If $u \in \omega$, we say that $a \in \omega$ is of type $(\boldsymbol{p} ; \boldsymbol{u})$ if

$$
x^{T} H(a) x \geqq x^{T} \Delta(u) x
$$

for all $x \in \phi_{\boldsymbol{R}}$ (real-valued sequences in $\left.\phi\right)$. Clearly, if $a$ is of type $(p ; u)$, then $a \in \omega_{+}$. If $a$ is of type $(p ; \delta)$, we say that $a$ is of type $\boldsymbol{p}$.

A simple generalization of the results of Lassner \& Uhlmann [20] proves that sequences of type $(p ; u)$ exist for any $u \in \omega$. However, such sequences tend to be highly divergent. For example, a simple manipulation of the techniques of Hofmann [15] yields the following result.

Proposition 4.14. If $(f(n))$ is a sequence of positive integers such that $f(0) \geqq 1$ and $f(n+1) \geqq 2 f(n)+n+2$ for $n \geqq 0$, then $\left(2^{f(n)}\right)$ is a sequence of type $p$.

Thus setting $f(n)=3^{n}$ or $f(n)=2^{n+2}-n-3$ gives us sequences of type $p$, but we do not know of any smaller ones. The practical use of sequences of these 
types lies in the following result.

Proposition 4-15. If $u \in \omega$, a is of type $(p ; u), g \in K(\phi)$ and $r \geqq 0$, then

$$
\sum_{n \geqq 0}\left|u_{n}\right|^{2} L_{r}^{n}(g)^{2} \leqq \sum_{n \geqq 0} a_{n} p_{r}^{(2 n)}\left(g_{2 n}\right) .
$$

The following fundamental result, leading to criteria for normality of the cone in $T(E, \lambda)$, uses the techniques of Schmüdgen [25].

Theorem 4-16. If, for any $u \in \lambda^{\times}$we can find $\alpha \in \omega_{+}$and $v \in \lambda^{\times}$such that

$$
p_{u, r}(g) \leqq \sum_{n \geqq 0} \alpha_{n} L_{r}^{n}(g)^{2} \leqq p_{v, r}(g)
$$

for $g \in K(\phi)$ and $r \geqq 0$, then $K(\lambda)$ is $t\left(\lambda^{\times}\right)$-normal.

Proof. For $u \in \lambda^{\times}$find $\alpha \in \omega_{+}$and $v \in \lambda^{\times}$as above, and let $V=|u|+$ $|v| \in \lambda^{\times}$. For any $w \in \lambda^{\times}$and $s \geqq 0$ we define $U_{w, s}=\left\{x \in T(E, \lambda): p_{w, s}(x) \leqq 1\right\}$ and we let $\left[U_{w, s}\right]=\left(U_{w, s}+K(\phi)\right) \cap\left(U_{w, s}-K(\phi)\right)$. If $x \in\left[U_{V, r}\right]$ we can write $x=y_{1}+g_{1}=y_{2}-g_{2}$ for $y_{1}, y_{2} \in U_{V, r}$ and $g_{1}, g_{2} \in K(\phi)$. Thus $p_{u, r}\left(g_{1}\right) \leqq p_{v, r}\left(g_{1}+g_{2}\right)$ $\leqq p_{V, r}\left(g_{1}+g_{2}\right)=p_{V, r}\left(y_{1}-y_{2}\right) \leqq 2$, and hence $p_{u, r}(x) \leqq p_{u, r}\left(y_{1}\right)+p_{u, r}\left(g_{1}\right) \leqq p_{V, r}\left(y_{1}\right)+$ $2 \leqq 3$. Thus $\left[U_{V, r}\right] \subseteq 3 U_{u, r}$, and so $K(\phi)$ is $t\left(\lambda^{\times}\right)$-normal.

We now consider some conditions on the sequence space $\lambda$ which guarantee that the conditions of Theorem $4 \cdot 16$ are satisfied.

Defimition 4.17. (a) $\lambda$ is said to be of type (N1) if, for any $u \in \lambda^{\times}$there exists $v \in \lambda^{\times}$such that $\left(v_{2 n}\right)$ is of type $(p ; u)$;

(b) $\lambda$ is said to be of type (N2) if, for any $u \in \lambda^{\times}$we can find $v \in k(\lambda)^{\prime}$ such that $\left|v_{n}\right| \geqq\left|u_{n}\right|$ for all $n \geqq 0$, and also find $w \in \lambda^{\times}$and $a$ of type $p$ such that $\left|w_{2 n}\right| \geqq a_{n}\left|u_{2 n}\right|$ for all $n \geqq 0$.

Theorem 4.18. If $\lambda$ is of type $(N 1)$ or $(N 2)$, then $K(\lambda)$ is $t\left(\lambda^{\times}\right)$-normal.

Proof. Suppose that $\lambda$ is of type (N1). For $u \in \lambda^{\times}$, choose $w \in \lambda^{\times}$such that $\left|u_{m+n}\right| \leqq\left|w_{m}\right|\left|w_{n}\right|$ for $m, n \geqq 0$. Choose $z \in \lambda^{\times}$and $\xi \in l_{2}$ with $\left|w_{n}\right| \leqq$ $\left|z_{n}\right|\left|\xi_{n}\right|$ for $n \geqq 0$ and $\|\xi\|_{2}=1$. Find $v \in \lambda^{\times}$such that $\left(v_{2 n}\right)$ is of type $(p ; z)$.

For any $g \in K(\phi)$ and $r \geqq 0$ we have

$$
\begin{aligned}
p_{u, r}(g) & \leqq \sum_{n \geqq 0}\left|u_{n}\right| \sum_{k=0}^{n} L_{r}^{k}(g) L_{r}^{n-k}(g) \leqq\left[\sum_{n \geqq 0}\left|w_{n}\right| L_{r}^{n}(g)\right]^{2} \leqq \sum_{n \geqq 0}\left|z_{n}\right|^{2} L_{r}^{n}(g)^{2} \\
& \leqq \sum_{n \geqq 0}\left|v_{2 n}\right| p_{r}^{(2 n)}\left(g_{2 n}\right) \leqq p_{v, r}(g)
\end{aligned}
$$

and so the result follows.

Suppose now that $\lambda$ is of type (N2). For $u \in \lambda^{\times}$, choose $z \in k(\lambda)^{\prime}$ such that 
$\left|z_{n}\right| \geqq\left|u_{n}\right|$ for $n \geqq 0$. Pick $w \in \lambda^{\times}$and $\xi \in l_{1}$ such that $\left|z_{2 n}\right| \leqq\left|\xi_{n}\right|\left|w_{2 n}\right|$ for $n \geqq 0$ and $\|\xi\|=1$. Pick $W \in k(\lambda)^{\prime}$ such that $\left|W_{n}\right| \geqq\left|w_{n}\right|$ for $n \geqq 0$, and finally choose $v \in \lambda^{\times}$and $a$ of type $p$ such that $\left|v_{2 n}\right| \geqq a_{n}\left|W_{2 n}\right|$ for $n \geqq 0$. For any $g \in K(\phi)$ and $r \geqq 0$ we have

$$
\begin{aligned}
p_{u, r}(g) & \leqq \sum_{n \geqq 0}\left|z_{n}\right| \sum_{k=0}^{n} L_{r}^{k}(g) L_{r}^{n-k}(g) \leqq \sum_{n \geqq 0} \sum_{k=0}^{n}\left|z_{2 k}\right|^{1 / 2} L_{r}^{k}(g)\left|z_{2 n-2 k}\right|^{1 / 2} L_{r}^{n-k}(g) \\
& \leqq\left[\sum_{n \geqq 0}\left|z_{2 n}\right|^{1 / 2} L_{r}^{n}(g)\right]^{2} \leqq \sum_{n \geqq 0}\left|w_{2 n}\right| L_{r}^{n}(g)^{2} \leqq \sum_{n \geqq 0}\left|W_{2 n}\right| L_{n}^{r}(g)^{2} .
\end{aligned}
$$

Since $W \in k(\lambda)^{\prime}$, the Cauchy-Schwarz inequality shows that the numbers $\left|W_{2 n}\right|^{1 / 2} L_{r}^{n}(g)$ and $\left|W_{n}\right| p_{r}^{(n)}\left(g_{n}\right)$ satisfy the same inequalities as do $L_{n}^{r}(g)$ and $p_{r}^{(n)}\left(g_{n}\right)$ in $(4 \cdot 7),(4 \cdot 8)$. Thus an adjusted Proposition $4 \cdot 15$ yields that

$$
p_{u, r}(g) \leqq \sum_{n \geqq 0}\left|W_{2 n}\right| L_{r}^{n}(g)^{2} \leqq \sum_{n \geqq 0} a_{n}\left|W_{2 n}\right| p_{r}^{(2 n)}\left(g_{2 n}\right) \leqq \sum_{n \geqq 0}\left|v_{2 n}\right| p_{r}^{(2 n)}\left(g_{2 n}\right) \leqq p_{v, r}(g),
$$

implying the result.

Finally, we notice that the criterion for normality given by Hofmann [12] also applies.

Definition 4-19. $\lambda$ is of type (N3) if for any $u \in \lambda^{\times}$we can find $v \in \lambda^{\times}$ such that:

(a) $\left|v_{n}\right| \geqq\left|u_{n}\right|$

$n \geqq 0 ;$

(b) $v_{2 n} \geqq\left[4(2 n-1) v_{2 n-1}\right]^{2} \quad n \geqq 1$;

(c) $v_{2 n+2} \geqq\left[8 n v_{2 n}\right]^{2} \quad n \geqq 1$.

Theorem 4-20. If $\lambda$ is of type $(N 3)$, then $K(\lambda)$ is $t\left(\lambda^{\times}\right)$-normal.

We notice that the other condition found in Hofmann [12] has been subsumed in the fact that $\left(\lambda, \tau\left(\lambda^{\times}\right)\right)$is nuclear.

The conditions (N1), (N2) and (N3) are all fairly similar, but do seem to be different. It should be noted that nontrivial regular tensor algebras with normal cone do exist. For example, if $\lambda$ is the countably echelon space defined by the family of steps $A=\{u(k): k \in N\}$, where $u(k)_{n}=2^{k^{n}}$, then $\lambda$ is of type $h$, of type $\left(N_{j}\right)$ for $j=1,2,3$, and $\left(\lambda, \tau\left(\lambda^{\times}\right)\right)$is a Fréchet convolution algebra. Thus $\left(T(E, \lambda), t\left(\lambda^{\times}\right)\right)$is a Fréchet regular tensor algebra with normal cone. Similarly, we can find other such spaces $\lambda$ by taking larger steps, so there certainly exist uncountably many regular tensor algebras with normal cones.

\section{§ 5. Open Questions}

An analysis could be made of the symmetric regular tensor algebra $T_{S}(E, \lambda)$, 
the quotient of $T(E, \lambda)$ by the kernel of the symmetrisation operator, particularly with regard to the moment and integral representation problems.

One the one hand, if $\pi$ is a representation of $T(E, \phi)$ arising from a state, then $\pi(x)$ is not generally essentially self-adjoint for symmetric $x$. On the other hand, if $T(E, \lambda)$ is a locally multiplicatively convex regular tensor algebra, then $\pi(x)$ is bounded, and hence essentially self-adjoint. It might turn out that there is a class of type $h$ spaces $\lambda$ such that representations are always standard; such a class would be interesting for applications.

Finally, we mention the perennial problem for applications of tensor algebras to quantum field theory-can we use the particular properties of $T(E, \lambda)$ to find a partical extension theorem for positive functionals? In particular, can we prove the existence of Wightman states of $T(\mathcal{S}(\mathbb{R}), \lambda)$ ?

\section{References}

[1] Alcantara, J., Order properties of a class of tensor algebras, Publ. RIMS Kyoto Univ., 18 (1982), 119-130.

[2] — Unpublished lecture notes, Göttingen University, 1982.

[3] —, A structure theorem for a class of locally convex *algebras, Ann. N.Y. Acad. Sci., 410 (1983), 71-73.

[4] Alcantara, J. and Dubin, D.A., I*-algebras and their applications, Publ. RIMS Kyoto Univ., 17 (1981), 179-199.

[5] Borchers, H.J., On the structure of the algebra of field operators, Nuovo Cimento 24, (1962), 214-236.

[6] - Algebraic aspects of Wightman field theory, in Statistical Mechanics and Field Theory, R.N. Sen and C. Weil (editors), Halsted Press, New York (1972)

[7] Brooks, R.M., On locally m-convex *-algebras, Pac. J. Math., 23 (1967), 5-23. 。

[8] Dixon, P.G., Generalied $B^{*}$-algebras, Proc. London Math. Soc. (3), 21 (1970), 693715.

[9] Gårding, L. and Wightman, A.S., Fields as operator-valued distributions in relativistic quantum field theory, Arkiv für Fysik, 28 (1964), 129-184.

[10] Garnir, H.G., deWilde, M. and Schmets, J., Analyse fonctionnelle. Tome III. Espaces fonctionnels usuels, Birkhäuser-Verlag, Basel (1973).

[11] Grothendieck, A., Produits tensoriels topologiques et espaces nucléaires, Mem. Amer. Math. Soc. 16, Providence, R.I. (1966).

[12] Hofmann, G., Ein hinreichendes Kriterium für die Normalität von Kegeln in Tensoralgebren und einige Anwendungen, Wiss. Z. KMU Leipzig, 27 (1978), 261-269.

[13] —. Topologien auf der Algebra der Testfunktionen der Quantenfeldtheorie, Wiss. Z. KMU Leipzig, $3 \mathbb{1}$ (1982), 35-42.

[14] —- Topologien auf Tensoralgebren, Wiss. Z. KMU Leipzig, 33 (1984), 16-24.

[15] - On the topological structure of tensor algebras and the closure of the cone of positive elements, Preprint KMU Leipzig (1985).

[16] Jarchow, H., Locally convex spaces, Teubner, Stuttgart (1981).

[17] Köthe, G., Topological vector spaces I, Springer-Verlag, Berlin (1969).

[18] Lassner, G., On the structure of the test function algebra, Dubna preprint JINR, E2-5254 (1970). 
[19] Lassner, G., Topological algebras of operators, Rep. Math. Phys., 3 (1972), 279-293.

[20] Lassner, G. aod Uhlmann, A., On positive functionals on algebras of test functions for quantum fields, Commun. Math. Phys., 7 (1968), 152-159.

[21] Peressini, A.L., Ordered topological vector spaces, Harper \& Row, New York (1967).

[22] Pietsch, A., Nuclear locally convex spaces, Springer-Verlag, Berlin (1971).

[23] Schaefer, H.H., Topological vector spaces, Springer-Verlag, Berlin (1971).

[24] Schmüdgen, K., The order structure of topological *-algebras of unbounded operators I, Rep. Math. Phys., 7 (1975), 215.

[25] — , Graded and filtrated topological *-algebras I. Graded normal topologies, Rep. Math. Phys., 18 (1980), 211-229.

[26] Streater, R.F. and Wightman, A.S., PCT, spin and statistics, and all that, W.A. Benjamin, New York (1964).

[27] Trèves, F., Topological vector spaces, distributions and kernels, Academic Press, London (1967).

[28] Uhlmann, A.. Über die Definitionen des Quantenfelder nach Wightman und Haag, Wiss. Z. KMU Leipzig, 11 (1962), 213-217.

[29] Wightman, A.S., Quantum field theory in terms of vacuum expectation values, Phys. Rev., 101 (1956), 860-866.

[30] Wyss, W., On Wightman's theory of quantised fields, Lectures in theoretical physics., University of Colorado, Boulder 1968, Gordon and Breach (1969).

[31] - The field algebra and its positive linear functionals, Commun. Math. Phys., 27 (1972), 223-234.

[32] Yngvason, J., On the algebra of test functions for field operators, Commun. Math. Phys., 34 (1973), 315-333. 
